\title{
Review Article \\ Status of LHC Searches for SUSY without R-Parity
}

\author{
Roberto Franceschini \\ CERN, Theory Division, Genève 23, 1211 Geneva, Switzerland \\ Correspondence should be addressed to Roberto Franceschini; franceschini.roberto@gmail.com
}

Received 26 March 2015; Revised 29 June 2015; Accepted 28 July 2015

Academic Editor: Michal Malinský

Copyright (C) 2015 Roberto Franceschini. This is an open access article distributed under the Creative Commons Attribution License, which permits unrestricted use, distribution, and reproduction in any medium, provided the original work is properly cited. The publication of this article was funded by SCOAP ${ }^{3}$.

In this contribution we briefly review the status of current searches for supersymmetry at the Large Hadron Collider, focusing especially on viable sub-TeV colored superpartners which can appear in nonstandard scenarios. The presented material covers mostly signals that do not crucially rely on the presence of large missing transverse momentum, with special emphasis on R-parity violating supersymmetry. For some scenarios the prospects for the next run of the Large Hadron Collider and future machines are also presented.

\section{Missing Transverse Momentum, Missing Supersymmetry}

Supersymmetry is the leading candidate for physics beyond the Standard Model of particle physics. It is currently subject to a very long list of experimental searches that try to use the high-energy collisions of the LHC beams to identify the production of new supersymmetric particles. So far the search for SUSY has generated a statistically [1] large number of papers in which any evidence of new physics has not been shown. Generically, searches for SUSY give bounds on the mass of colored superpartners [2]. Consider

$$
M_{\text {SUSY }}>1 \mathrm{TeV} \text {. }
$$

In this contribution, we review the qualitative aspects of the searches that have been carried out by the experiments and the possible consequences of these results for our picture of supersymmetry as the theory for physics at the TeV scale.

Searches for new physics are usually conducted by searching for final states containing some combination of

$$
\text { jets, leptons, photons, and mET. }
$$

Jets, leptons, and photons are, roughly speaking, the measurable objects that are devised to capture the concepts of an energetic quark or gluon, an isolated electron or muon, and a nonvirtual photon that are used to describe highenergy scattering in terms of a Lagrangian. $\mathrm{mET}$ is, instead, a nonmeasurable object that is defined "by contrast" as the imbalance of momentum in the plane orthogonal to the colliding beams. Since momentum is known to be conserved to very high accuracy, we think that $\mathrm{mET}$ is a consequence of the production of particles that do not interact with the detector materials. One example of a source of mET is indeed the neutrino, which was originally observed as a violation of momentum conservation in radioactive decays. As the neutrino history demonstrates, the presence of $\mathrm{mET}$ above the expected level due to detector imperfections is quite noticeable and immediately suggests the production of particles experiencing only weak interactions [3]. In the Standard Model the only noninstrumental source of $\mathrm{mET}$ is the production of neutrinos, which is not very abundant when compared to most other processes, especially when involving strong interactions. For this reason a signal with $\mathrm{mET}$ is quite easy to spot over backgrounds, which is why mET is in bold typeface in (2). Because of this experimentally striking nature, most experiments looking for SUSY at particle colliders search for large mET signals. Furthermore, these large mET signals are also predicted by most supersymmetric models, especially those that can provide a Dark Matter candidate. Building on these experimental and theoretical arguments, all large mET searches build their strength (and their weakness at the same time) on the fact that in the production of supersymmetric particles one forcedly produces also purely weakly interacting stable particles that give rise to mET. For these reasons we 
can (somewhat provocatively) dub the present searches for supersymmetry as "searches for supersymmetric Dark Matter in the decay of colored superpartners."

While this summary of the scope of the present searches might be a bit too hasty, it renders well the actual reach of the present results from the LHC. In fact for most searches if one considers signal cross sections lower than those of colored particles the mass reach quickly vanishes. Similarly as one reduces the amount of missing transverse momentum in the signal events the mass reach quickly drops as well. These two axes, reduction of cross section and reduction of missing transverse momentum, are the two main handles that are typically involved when light supersymmetric particles evade present bounds.

\section{Sub-TeV Colored Superpartners}

The stringent bounds that emerge from the experimental results of the first run of the LHC are certainly a motivation, and a valuable chance, to reconsider our motivation for SUSY at the TeV scale as well as our approach to the search of its signals.

A common element of the vast majority of searches is the fact that in each new physics event containing supersymmetric particles the scattering reaction has to be of the type

$$
p p \longrightarrow \mathrm{SUSY} \longrightarrow \chi \chi+\mathrm{SM},
$$

where $\chi$ denotes a generic weakly interacting massive particle that gives rise to $\mathrm{mET}$. Of course if the intermediate supersymmetric states that mediate reaction equation (3) have reduced cross section, their search will be more difficult and the constraints from present searches would be looser. In this contribution we do not consider this type of ways out of the current bounds from the LHC. These situations with reduced cross section for the new physics colored states are typical in models with Dirac Gauginos, which are covered elsewhere in this volume. Instead, we want to consider the possibility of different types of SUSY models where the typical signal does not feature sources of mET beyond those of the SM, that is, in reactions of the type

$$
\mathrm{pp} \longrightarrow \mathrm{SUSY} \longrightarrow \mathrm{SM}
$$

These two scatterings in (3) and (4) differ most importantly in the amount of mET that they generate. In fact in the case of scatterings of type equation (4) no sources of mET beyond the Standard Model are expected. At the level of theoretical description, the difference between SUSY models giving rise to reactions of type equations (3) and (4) is in the amount of discrete symmetries that one imposes on the model. Therefore the exploration of both types of signals is needed as it is very informative about the symmetries of the new physics. The symmetry structure of the two classes of models differs in the aspects outlined in what follows. Taking the Lagrangian of the Standard Model and extending it to respect SUSY one would obtain the following gauge invariant superpotential interactions in addition to the usual MSSM superpotential:

$$
W_{\mathrm{RPV}}=\lambda^{\prime \prime} u^{c} d^{c} d^{c}+\lambda^{\prime} L Q d^{c}+\lambda L L e^{c}+\mu^{\prime} L H_{u} .
$$

These interactions violate baryon number $\left(\lambda^{\prime \prime}\right)$ or lepton number $\left(\lambda, \lambda^{\prime}\right.$, and $\left.\mu^{\prime}\right)$. Without advocating a specific origin of these couplings one expects that, once R-parity is broken, all the interactions are generated and both baryon number and lepton number are violated, which is very dangerous in view of the stringent bounds on the conservation of these quantities [21]. The extension of the Standard Model to respect supersymmetry ends up altering the accidental symmetries of the Standard Model through these new Yukawa interactions and mass mixings. In the past, these new interactions have mostly been regarded as a source of trouble, as they make it hard for the model to not be in contrast with experiments. For this reason, a symmetry called R-parity has been put by hand in the model that we usually call Minimal Supersymmetric Standard Model (MSSM). R-parity, killing the interactions in (5), forbids the lightest superpartner to decay and hence provides a stable particle which, if weakly interacting, might be a Dark Matter candidate. (Although a weakly interacting particle of mass around the Fermi scale is no longer available as a Dark Matter candidate in RPV models, it is worth mentioning that other Dark Matter candidates exist in these models. One example is the decaying gravitino found in bilinear RPV scenarios, where $\mu^{\prime}$ is the only nonzero RPV coupling $[22,23]$. One other example is a stable gravitino in models of baryonic RPV, where only $\lambda^{\prime \prime}$ is nonzero. This gravitino would follow the same dynamics of the usual R-parity conserving gravitino [24] and might easily be heavy enough to suppress proton decay below the current limits $[25,26]$. Furthermore axions and particles related to it through supersymmetry could be Dark Matter candidates as well.) This particle is $\chi$ in the above equation and is the source of $\mathrm{mET}$ around which the vast experimental program for SUSY searches has been built.

Taking a step back in the path that leads from SUSY to mET and Dark Matter we can choose to cope with the new interactions in (5) in a different way. In Section 2.2.1, we discuss which theoretical ideas could provide an approximate symmetry that puts these interactions under theoretical control, still not putting them to zero. In Section 2.2 we explore the observable consequences that a nonvanishing coupling in (5) would have at the LHC experiments.

2.1. Stealth and Compressed SUSY. In some cases reactions equations (3) and (4) might be very hard to distinguish, because of the elusive nature of the $\chi$ particles. As invisible particles they can only be observed by "contrast" looking at the entire set of particles produced in the reaction and imposing on them some kind of momentum conservation law. This means that every time that $\chi$ does not carry large momentum it might be very difficult to observe the effects of its production.

A typical case where $\chi$ gives less observable signals than the ones targeted in standard searches is "stealth" supersymmetry. To understand what it is, we look at the kinematics of a two-body decay. Consider

$$
A \longrightarrow b c
$$


Conservation of 4-momentum implies that in the rest frame of the parent particle

$$
\begin{aligned}
& E_{b}=\frac{m_{A}^{2}-m_{c}^{2}+m_{b}^{2}}{2 m_{A}}, \\
& E_{c}=\frac{m_{A}^{2}+m_{c}^{2}-m_{b}^{2}}{2 m_{A}},
\end{aligned}
$$

which is the usual monochromatic energy of a two-body decay product in the rest frame of the decaying particle. The same conservation of 4-momentum can also be rewritten in the form of two opposite 3 -momenta $\vec{p}_{b}=-\vec{p}_{c}$ with equal magnitudes. One has

$$
p_{c}=p_{b}=\frac{M_{A}}{2} \sqrt{\lambda\left(1, \frac{m_{b}^{2}}{m_{A}^{2}}, \frac{m_{c}^{2}}{m_{A}^{2}}\right)}
$$

where $\lambda(x, y, z)=x^{2}+y^{2}+z^{2}-2 x y-2 x z-2 y z$ is a measure of the available phase-space of the decay. As can be seen from these simple kinematical considerations, when the decay happens close to a degeneracy $m_{A} \simeq m_{b}+m_{c}$ the 3 momenta of the particles $b$ and $c$ are suppressed. Consider

$$
\begin{aligned}
& p_{b} \simeq\left(M_{b}, 0\right) \\
& p_{c} \simeq\left(M_{c}, 0\right) .
\end{aligned}
$$

This situation arises when the spectrum is "compressed"; that is, the masses are almost too heavy to close the phase-space of the decay. In general this situation gives rise to small 3momenta particle $A$ rest frame, but 3-momenta may be large once the decay is observed in a frame with a large enough boost from the $A$ rest frame. This means that if a new physics signal generically gives little mET because of "compression" of the spectrum, one can in principle try to observe events with larger boosts in order to increase the amount of mET observed in the events. However, there are cases in which changing frame does not help. In fact, if one of the two particles is more massive than the other all the energy of the decaying particle $A$ is transferred to the mass of the heavy particle, whereas the momenta of the decay products are comparably smaller. Consider

$$
\begin{aligned}
p_{\text {heavy }} & =\{M+O(\epsilon), \epsilon\} \\
p_{\text {light }} & =\{\epsilon, \epsilon\} .
\end{aligned}
$$

In this situation, the emission of the light particle in the decay can be pictured as almost unnoticeable soft emission in the conversion of the decaying particle into the heavy daughter particle. Although we derived the kinematics of this decay in the rest frame of $A$, the latter statement holds as well in the laboratory frame, as the Lorentz transformation of $p_{\text {light }}$ will again give a vector of negligible momentum compared to the Lorentz transformation of $p_{\text {heavy }}$.

The presence of a light invisible particle in the spectrum can be achieved relatively easily [27], for instance, in scenarios of gauge mediated supersymmetry breaking or any scenario with low scale of mediation of supersymmetry breaking where the gravitino can be much lighter than the other superpartners or in scenarios in which the lightest neutralino is nearly massless. In these circumstances, due to its lightness, it is very hard to tell apart events where $\chi$ is present from those where it is not. For this reason, it is very difficult to reject Standard Model events and retain new physics ones on the basis of the measured missing transverse momentum. In these cases, it is also difficult to understand whether the new physics reaction belongs to type equations (3) and (4). However, as we discuss in the next section, (4) can be identified thanks to other distinctive features, such as the presence of new directly measurable resonances in the new physics event.

2.2. R-Parity Violation. As explained above, $\mathrm{mET}$ is a very powerful discriminator of physics beyond the Standard Model, due to the relatively low rates of events produced by Standard Model physics with large mET. A similarly powerful discriminator of new physics events is electrons and muons that are at the same time relatively infrequent final states in Standard Model physics and are also very clean to measure. This implies that when one or more among the couplings $\lambda, \lambda^{\prime}, \mu^{\prime}$ in (5) are nonzero, the lightest superpartner can decay through these interactions. In general, the decay of the lightest superpartner through these interactions will systematically produce charged leptons as these couplings break lepton number. The result is that when the lepton number violating couplings are important for the decay of the lightest superpartner, new physics events give rise to processes of the type

$$
p p \longrightarrow \text { SUSY } \longrightarrow \text { many leptons }+X
$$

For these processes we have very stringent bounds [2] and generically the masses of the superpartners end up being constrained to be at the $\mathrm{TeV}$ scale or higher. In view of these bounds from leptonic final states, lepton number violating couplings are of no help if one is trying to explain why subTeV SUSY has not been observed in experiments at the $\mathrm{TeV}$ scale (see, e.g., [28] for possible exceptions).

A more intriguing possibility is given by the baryon number violating coupling

$$
\lambda_{i j k}^{\prime \prime} \cdot u_{i}^{c} d_{j}^{c} d_{k}^{c}
$$

which breaks baryon number but conserves leptons number and per se does not mediate proton decay. When this coupling dominates the decay of the lightest superpartner, we expect purely hadronic final states to appear from the production of new supersymmetric states. One has

$$
p p \longrightarrow \text { SUSY } \longrightarrow \text { hadrons. }
$$

These final states are very frequent in Standard Model scatterings, owing to the hadronic nature of the initial state of the LHC collisions and to the strength of hadronic interactions. Therefore the discovery of new physics in this type of final states is considerably more challenging than in most other final states. This is true even after taking into account that the absence of sources of mET from physics beyond the Standard 
Model opens the possibility of searching for new states through resonance searches. A nice example that displays this fact is squark production. Consider

$$
\begin{aligned}
& p p \longrightarrow \widetilde{q} \widetilde{q} \longrightarrow q q \chi \chi \text { (R-parity Conserving) } \\
& \text { versus } p p \longrightarrow \widetilde{q} \widetilde{q} \longrightarrow q 9 q q \quad \text { (R-parity Violating). }
\end{aligned}
$$

Despite the possibility of reconstructing a resonance from the decay $\tilde{q} \rightarrow q q$, the RPV scenario is very loosely bounded, especially for the most interesting mass range $m_{\tilde{q}} \sim 100 \mathrm{GeV}$ $[5,29]$. If one is interested in just heavy flavor squark states, as a naturalness argument would want them to be the lightest squarks, then no bound exists for $m_{\tilde{t}}>100 \mathrm{GeV}$. The stop squark example shows very clearly that sub-TeV superpartners are still allowed when R-parity is not imposed on the MSSM. However it must be stressed that baryonic RPV SUSY has some interesting bounds from the LHC. For instance, the searches for objects such as the gluino can exploit the richer and more structured final state that originates from the gluino decay, which produces a multijet resonance. This search has been the subject of several interesting experimental and theoretical developments in the recent times. For instance, the possibility to use jet-substructure techniques to deal with multijet signals that appear in a final state of unknown (and in general not fixed) multiplicity from the gluino decay has been explored in [15]. These techniques might also be useful to reach the very faint signals that would originate from squark decay as in (14) [30]. Another front of recent progress has to do with the use of heavy flavor tagging in multijet searches. In fact, most theories that explain the origin of the RPV couplings predict peculiar dependences of the couplings on the flavor of the quark. These predictions [25, 31-34] (see also [25] for exceptions to the MFV prediction) tend to favor heavy flavor final states and certain searches for RPV SUSY are already covering the space of possible flavor signatures of the different models [11]. In this case the information about the presence of heavy flavor final states in the decay of new physics particles can make the difference between attaining a discovery and a bound on the faint signals of light RPV stops [35].

2.2.1. Origin of the RPV Couplings. When R-parity is not imposed, one needs to find a dynamical mechanism that makes the RPV couplings small enough to avoid excessive baryon or lepton number violation. Barring the option of just assuming that these couplings are small by accident (although this "feature" would be preserved by nonrenormalization of the superpotential), one interesting possibility to control the size of the RPV couplings is to associate them with the breaking of the flavor symmetries of the Standard Model. In fact if one takes the masses of the fermions of the Standard Model and put them to zero, then the Standard Model Lagrangian acquires a large flavor symmetry that redefines, for instance, the flavor up, charm, and top of the right handed up-type quark fields. Similar symmetries exist for all the fermions of the Standard Model and overall there is a

$$
\begin{gathered}
S U(3)^{6}=S U(3)_{\mathrm{Q}} \otimes S U(3)_{u} \otimes S U(3)_{d} \otimes S U(3)_{L} \\
\otimes S U(3)_{e} \otimes S U(3)_{v}
\end{gathered}
$$

symmetry that one can impose on the supersymmetric extensions of the Standard Model and that would make all the interactions of $(5)[31,32]$ vanish. In this way, the size of the RPV couplings is connected to the breaking of the flavor symmetry and hence to the masses of the fermions that are involved in the interaction. The generic prediction of complete models is

$$
\lambda_{i j k}^{\prime \prime} \sim f_{\mathrm{CKM}} \cdot\left(\frac{m_{u_{i}} m_{d_{j}} m_{d_{k}}}{m_{t}^{3}}\right)^{\alpha},
$$

where $f_{\mathrm{CKM}}$ is a factor from the CKM matrix that tends to suppress couplings involving different generations of quarks and $\alpha=1$ in the most simple models but $\alpha<1$ is attainable as well [25]. The predictions of these models, especially the minimal models where $\alpha=1$, tend to favor the production of heavy quark jets. In light of these predictions the experimental exploration [11,35] of flavored final states from the decay of RPV SUSY states is certainly very motivated.

\section{Current Searches and Prospects for the LHC 14 TeV Run and Beyond}

The presence of a stable invisible particle at the bottom of each decay chain of supersymmetric particles is the very reason for large mET signals expected in supersymmetric models. The large mET is usually a powerful discriminator to reject backgrounds; however, in some occasions other features of the signal can be helpful as well. For instance, in some cases we can derive from first principles an approximate shape of the distribution of some observable. A classic example is the shape of a Breit-Wigner resonance which can be effectively searched over a smoothly falling background. This has been the case, for instance, of the observation of the Higgs boson decay into two photons (up to the fact that due to resolution effects the shape is actually closer to a gaussian than to a Breit-Wigner).

Supersymmetric models with stable invisible particles unfortunately do not usually benefit from this type of searches because the invisible particles carry away momentum and it is not possible to use them in the reconstruction of the BreitWigner resonance. In presence of invisible particles, it is still possible to exploit features of multiparticles invariant masses [36-38] or single particle properties [39, 40]. However, such methods are useful only in searches for specific scenarios [41, 42] and are in general more suited to probe the mass spectrum of the model rather than to isolate a signal from the backgrounds. A more systematic use of resonant features in supersymmetric model requires no invisible particles in the final state of the decays.

As discussed above, invisible supersymmetric particle arises only as a consequence of having imposed R-parity in the MSSM. Without this feature of the model, new interactions are present; they are Yukawa couplings of the type in (5) and in general they can mediate the decay of any superpartner into a set of states of the Standard Model; for instance,

$$
\begin{gathered}
\tilde{q} \longrightarrow j j \\
\text { or } \tilde{g} \longrightarrow q \tilde{q} \longrightarrow q \bar{q} q .
\end{gathered}
$$




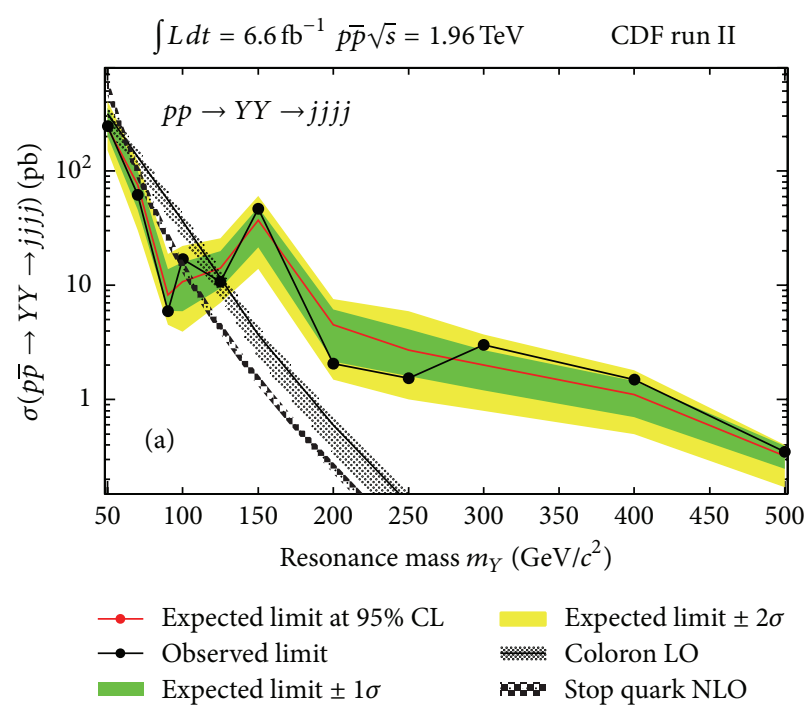

(a)

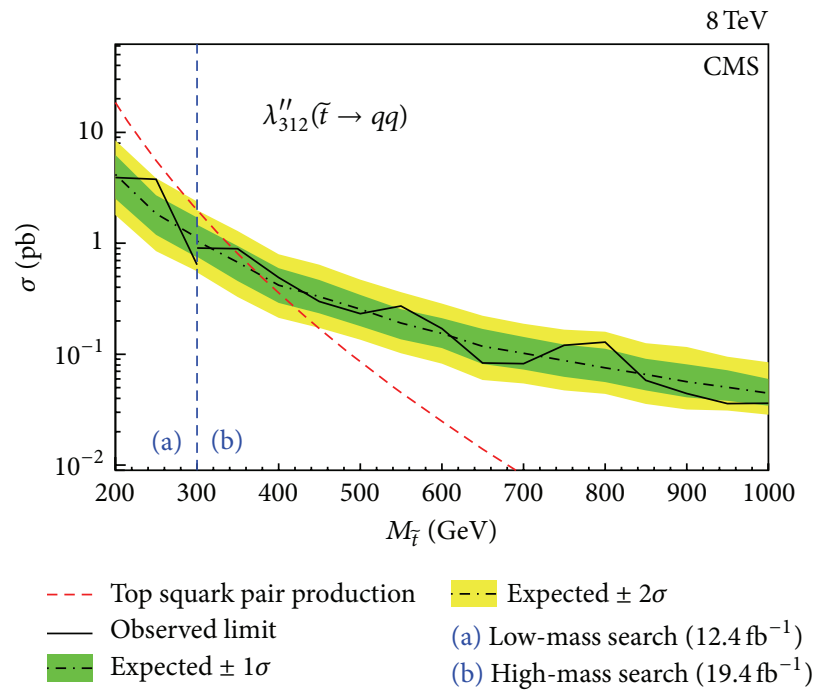

(b)

FIGURE 1: Limits on paired dijet production confronted with the production cross section of a single stop quark at the TeVatron [4] (a) and at the LHC [5] (b). As indicated in the legend, the black solid line represents the excluded cross section for the new physics reaction as a function of the mass of the new state. The green and yellow bands are the total $1 \sigma$ and $2 \sigma$ uncertainties on the exclusion. The predicted cross section for the new state is indicated by a solid line per each specific new physics model. The model is excluded for each mass choice for which the predicted cross section is above the exclusion.

These couplings in general have to be small because they can mediate baryon number or lepton number violating process such as proton decay or oscillation between neutron and antineutron or mediate flavor changing neutral currents processes. Despite their smallness they are crucial to avoid invisible stable particles in the model. In fact, RPV coupling of order $10^{-5}$ would still be large enough to give a decay $\chi \rightarrow$ $q \bar{q} q$ with average decay path below $10^{-6}$ meters. In what follows we discuss several simplified models for RPV supersymmetry that give rise to resonances, roughly in the order from lowest multiplicity to higher multiplicity. We concentrate on baryonic RPV signals, as leptonic RPV is usually very tightly bounded by the presence of hard leptons in the final states $[43,44]$ (scenarios of leptonic RPV where this statement might not be true have been studied in [28]).

3.1. The (Heavy Flavor) RPV Squark Simplified Model. In this simplified model, the only light supersymmetric particle that can be produced via QCD interaction is a squark (see Table 1).

For this simplified model, the signal consists of 4 jets in which one can find a resonance from $\tilde{q} \rightarrow j j$. This search is quite challenging as it faces a large QCD background from multijet events. The 2010 run of the LHC at low instantaneous luminosity has been very helpful to probe this type of simplified models as it has provided data with lower trigger thresholds and lower QCD background rates. Together with recent CDF results [4], the ATLAS search [45] provides the best limits of this type of processes at low mass. Recent bounds from high instantaneous luminosity data have been put and constrain squarks from $200 \mathrm{GeV}$ to $350 \mathrm{GeV}$ [5]. The full collection of limits from this search is reported in Figure 1.
TABLE 1

\begin{tabular}{llc}
\hline BSM particles & Production & Decay \\
\hline$\widetilde{q}$ & $p p \rightarrow \widetilde{q} \widetilde{q}^{*}$ & $\widetilde{q} \rightarrow j j$ \\
\hline
\end{tabular}

For a single squark the limits so far exclude masses up to $100 \mathrm{GeV}$.

The projection for the exclusion of this simplified model at the LHC Run 2 and High Luminosity LHC have been studied in [6], which finds that masses from $300 \mathrm{GeV}$ to about $1 \mathrm{TeV}$ can be excluded at the end of High Luminosity Run. For a reliable exclusion at low mass, special care is needed as the backgrounds become larger and more uncertain. In [30], the possibility to exploit the lightness of the squarks to search for the production of highly boosted squarks using jet-substructure techniques has been studied. The result is reported in Figure 2 for the expected exclusion if such analysis would be run on $8 \mathrm{TeV}$ LHC data. The estimated exclusion extends up to $150 \mathrm{GeV}$. Given the nature of this search it is expected to improve as higher energy machines are available, as the production of boosted stops will become more abundant.

If the squark is a heavy flavor, more special signals are expected to arise; for instance,

$$
\begin{aligned}
\tilde{t} & \longrightarrow b j \\
\text { or } \tilde{b} & \longrightarrow t j .
\end{aligned}
$$

The presence of bottom or top quarks in the final state is a powerful handle to discriminate these signals from the background and recently bounds have been obtained [5] 


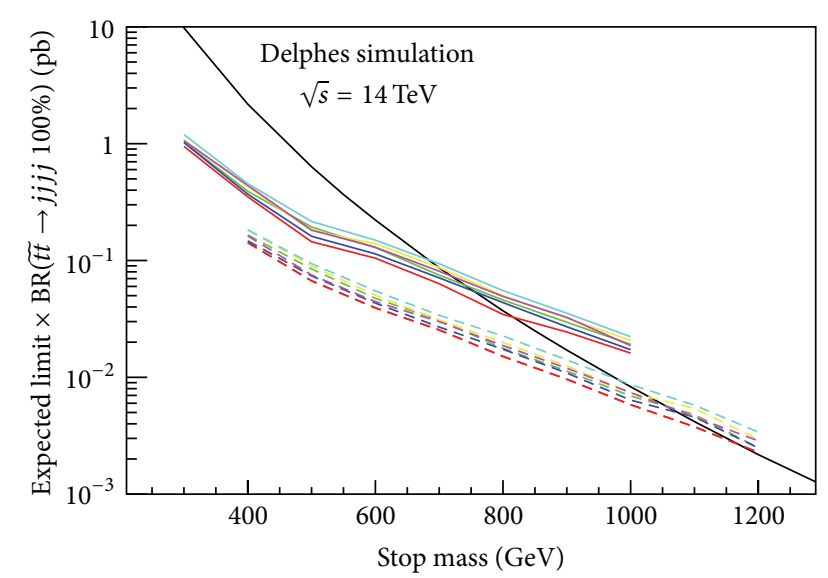

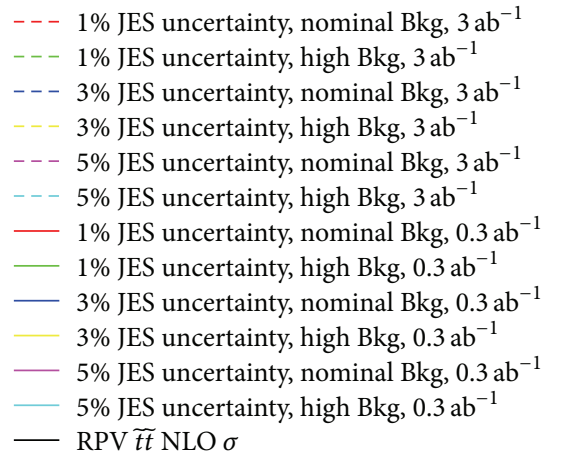

FIgURE 2: Projected exclusion reach for the LHC Run 2 and beyond [6] for the RPV squark simplified model. In this picture the many colored lines represent the expected exclusion under a given assumption of Jet Energy Scale (JES) uncertainty and background systematics. For more details on this assumption, we refer to the original work [6].

exploiting the presence of heavy flavors in the final state. The bounds are reported in Figure 3 and show an exclusion between $200 \mathrm{GeV}$ and $385 \mathrm{GeV}$. The region between $100 \mathrm{GeV}$ and $200 \mathrm{GeV}$ is presently not probed by LHC, due to the large thresholds needed for trigger and for QCD background rejection. With an analysis targeted to this region, discovery should be possible from the CDF bound at $100 \mathrm{GeV}$ up to masses around $200 \mathrm{GeV}$ using LHC Run 1 data [30, 35]. Higher energy colliders will be able to extend the discovery reach beyond $200 \mathrm{GeV}$, as the limitations in this channel arise mainly from the stop production cross section and not from the poor knowledge of the background.

3.2. The (Heavy Flavor) RPV Gluino Simplified Model. In this simplified model, only the gluino is light enough to be produced in $p p$ collisions. The gluino can decay only through a three-body decay into three fermions, mediated by a virtual squark. Depending on the mass of the virtual squark, this might give rise to displaced vertexes, but here we will consider the case of prompt decay, which is usually the case for squarks masses below $1 \mathrm{TeV}$ (see Table 2).

Searches for resonances decaying into 3 light flavor jets have been conducted at both ATLAS and CMS. For the case in which the 3 jets are resolved, limits are reported in Figure 4,

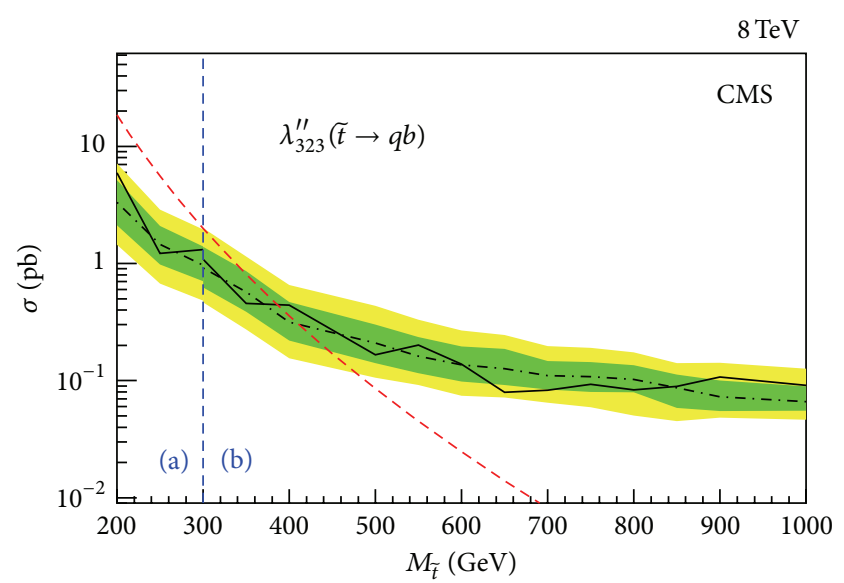

$\begin{array}{ll}--- \text { Top squark pair production } & \text {-.- Expected } \pm 2 \sigma \\ - \text { Observed limit } & \text { (a) Low-mass search }\left(12.4 \mathrm{fb}^{-1}\right) \\ -- \text { Expected } \pm 1 \sigma & \text { (b) High-mass search }\left(19.4 \mathrm{fb}^{-1}\right)\end{array}$

FIgURE 3: Exclusions on the RPV heavy flavor squark simplified model from the $8 \mathrm{TeV}$ run of the LHC [5]. Color codes are the same as in Figure 1.

TABLE 2

\begin{tabular}{lcc}
\hline BSM particles & Production & Decay \\
\hline$\widetilde{g}$ & $p p \rightarrow \widetilde{g} \widetilde{g}$ & $\widetilde{g} \rightarrow j j j$ \\
\hline
\end{tabular}

TABLE 3

\begin{tabular}{lcc}
\hline BSM particles & Production & Decay \\
\hline$\widetilde{g}$ & $p p \rightarrow \widetilde{g} \widetilde{g}$ & $\tilde{g} \rightarrow J_{u} J_{d} J_{d}$, \\
& where $J_{u}=\{t, c, j\}$ and $J_{d}=\{b, j\}$ \\
\hline
\end{tabular}

which shows a bound up to about $150 \mathrm{GeV}$ [7] and from 200 to about $800 \mathrm{GeV}$ [8-10].

If one of the virtual squarks that mediates the gluino decay has a preference for coupling to a certain flavor of quarks the previous simplified model will not capture this feature, which, as a matter of fact, is rather well justified in concrete models of RPV supersymmetry. To capture this possibility, a slightly different simplified model can be considered with gluino decays to heavy flavors, in suitable flavor combinations $\widetilde{g} \rightarrow J_{u} J_{d} J_{d}$, where $J_{u}=\{t, c, j\}$ and $J_{d}=\{b, j\}$ (see Table 3 ).

For example, ATLAS puts a bound [46] from $500 \mathrm{GeV}$ to about $1 \mathrm{TeV}$ on the flavor combination $\widetilde{g} \rightarrow t b j$. A more extensive exploration of the flavor structure of the $J_{u} J_{d} J_{d}$ final state is carried out in [11]. The highest excluded mass varies depending on the flavor combination assumed for the $J_{u} J_{d} J_{d}$ final state. In Figure 5, we report the result for zero charm quarks in the plane of bottom and top branching fraction.

Also for this simplified model, as it was the case for the RPV squark simplified model, it is particularly tough to put bounds on light particles. For instance, in [10], the lowest mass for which it is possible to put a limit is about $400 \mathrm{GeV}$, because of the trigger thresholds. The limitations arise because the searches try to identify a bump from the $j j j$ resonance over a smooth background from QCD multijet 

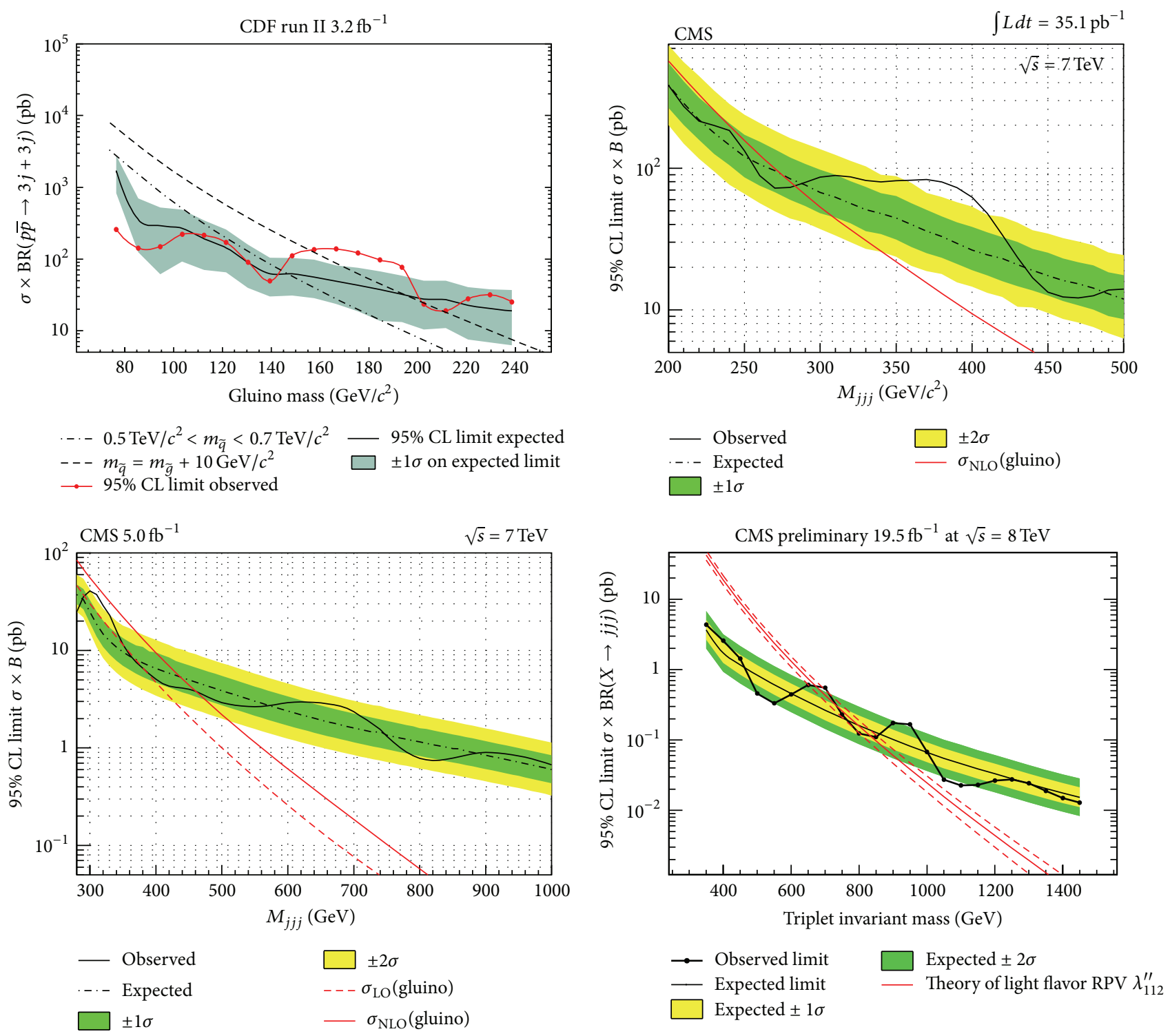

FIGURE 4: Limits on the gluino RPV simplified model for the resolved jet final state [7-10]. Color codes are the same as in Figure 1.

production. To overcome this restriction, searches that compare counted events with theory of Monte Carlo predictions have been performed. These "cut and count" searches are in general not requiring to see a bump or any feature in the spectrum of multijets events. To improve the robustness of the search, they normally use data to normalize the expected number of events in presence of pure background. Nevertheless, they rely more than bump searches on the theoretical prediction of the backgrounds, which makes it more challenging to obtain reliable bounds. Results from this cut and count search [15] are reported in Figure 9, where exclusions from $100 \mathrm{GeV}$ upwards are shown. It is remarkable that light gluinos cross sections are excluded by a large factor, which should guarantee the exclusion even in presence of large uncertainties.

It has been pointed out that for RPV Majorana gluino decaying into heavy flavors it is possible to have a signal with two hard leptons of equal electric charge [47]. Limits from this signal have been obtained from the $8 \mathrm{TeV}$ run of the LHC and exclude masses up to $900 \mathrm{GeV}$ [12]. Estimates for the $14 \mathrm{TeV}$ LHC have been presented in [13] and are reported in Figure 6. From this analysis, the High Luminosity LHC should be able to discover a gluino of mass up to $1.6 \mathrm{TeV}$.

3.3. High-Multiplicity Resonances Simplified Models. Spectra just slightly richer than those considered above usually give rise to several jets in the final state of the RPV supersymmetric event. A simple and motivated variation of the above models is the addition of a light neutralino in the simplified model. The stop simplified model, for which the search is so difficult at hadronic machines (see Figure 1), now decays via ordinary MSSM couplings into a neutralino, which in turn decays to three jets (see Table 4). 
TABLE 4

\begin{tabular}{lcc}
\hline BSM particles & Production & Decay \\
\hline$\tilde{t}, \chi$ & $p p \rightarrow \widetilde{t t}^{*}$ & $\tilde{t} \rightarrow t \chi, \chi \rightarrow j j j$ \\
\hline
\end{tabular}

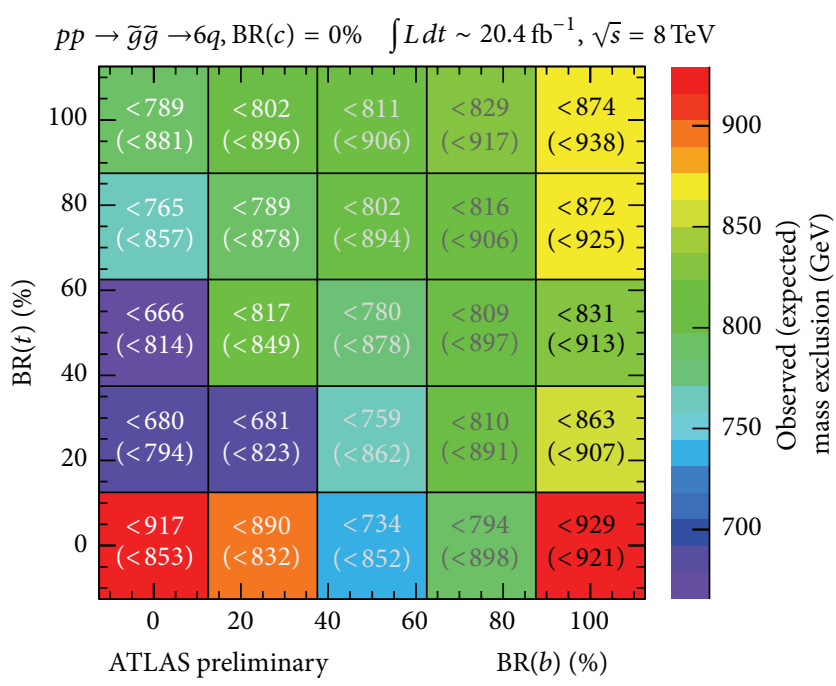

FIGURE 5: Highest mass excluded at 95\% CL in the search for heavy flavor three-body decay of gluino in RPV supersymmetry [11]. Vertical and horizontal axes indicate the branching fraction into $b$ and $t$ quarks and the colored boxes indicate the corresponding highest excluded mass, as indicated by the color coded legend as well.

Considering this high-multiplicity final state, the discovery reach is much improved. We report in Figure 7 the estimated discovery and exclusion reach at the High Luminosity LHC for this simplified model. Masses up to $1.5 \mathrm{TeV}$ will be excluded by the High Luminosity LHC and discovery up to $1 \mathrm{TeV}$ will be possible.

Considering in full generality the possible signals that arise in RPV simplified model one is immediately led to consider a large number of possible final states. Some example of spectra that gives rise to large multiplicity is shown in Figure 8 . The spectra in the figure are particularly difficult to discover because of the heaviness of third generation squarks, which gives no top quark final states. Explicit searches have been conducted up 8-jet final state [48] at CMS and up to 7 jets at ATLAS [11]. For higher multiplicities, the typical search for nonperturbative phenomena such as black-hole formation [49] is able to capture signal from complex RPV spectra. The interpretation of these searches in terms of RPV simplified models has been studied in [14]. The result of this recast of ATLAS [11] and CMS [49] analyses gives exclusions of gluino masses up to $1 \mathrm{TeV}$.

In [6], the reach for these more complex simplified models for $p p$ colliders up to $33 \mathrm{TeV}$ has been explored. The summary of these studies is reported in Table 5.

For these more general final states, it becomes harder to have reliable predictions for many jets backgrounds. Furthermore when a light supersymmetric particle decays into many partons it might be hard to resolve each single parton into a jet. When jets are lost, it also becomes harder to reconstruct resonances as some momentum is not measured. Additionally, if one insists on getting a large number of jets from light particles, then search for a signal in a scarcely populated region of its own phase-space might be needed, that is, in a tail of the signals kinematic distributions. In this case, it is usually harder to have a reliable theoretical prediction of the cross section of the signal in this particular region of phase-space. To overcome these difficulties, the use of jet-substructure techniques has been considered to try to reconstruct resonances in a large radius jet [50-52]. ATLAS has applied this type of ideas to the search of gluinos decaying into three jets $\tilde{g} \rightarrow j j j$ and has probed mass as low as $100 \mathrm{GeV}$ both with a "skinny" jet analysis and with a "fat" jet analysis. The results are reported in Figure 9. At the $7 \mathrm{TeV}$ LHC, the performance of the boosted strategy is comparable to the traditional search for low gluino mass. However, this search serves as a first step to validate the fat jet technique, which is expected to become more and more relevant as machines of higher energy become available.

3.4. Displaced RPV Supersymmetry. The RPV couplings are often bounded to be very small to avoid limits on proton decay, neutron-antineutron oscillations, and other limits [21]. For this reason, it is expected that these couplings must be so small that they can result in metastable particles whose decay length can be observed in the detectors, which is usually possible for average decay paths in the range from $0.01 \mathrm{~cm}$ to $10 \mathrm{~m}$.

A variety of signals can arise in this scenario [53]. Most of them would easily escape standard searches for new physics, which focus on prompt production and decay of the new particles.

Among the many possible signals explicit bounds from the experiments exist for the squark-neutralino simplified model and for the squark LSP simplified model. In some scenarios, recast bounds [19] are available as well; however, most of them rely on delicate secondary vertex reconstruction, whose efficiency is nontrivial to carry from one model to another. Nevertheless, these recasts fill an important gap in the current searches. Therefore, they are an important element for the current status of searches for displaced signals from RPV supersymmetry.

3.4.1. The Displaced RPV Squark-Neutralino Simplified Model. In the squark-neutralino simplified model, squarks are produced and then decay to a neutralino, whose decay is displaced, and give rise to jets or leptons, or both, depending on the RPV couplings that are assumed (see Table 6).

At present, only bounds for LQD [16, 17] and LLE [18] R-parity violating interactions are given in this simplified model. The bounds are reported in Figure 10 and they usually rule out squarks below about $1 \mathrm{TeV}$ for a large range of detector-size displaced vertexes.

3.4.2. The Displaced RPV Neutralino Simplified Model. This simplified model is obtained from the previous squarkneutralino simplified model when squarks are decoupled (see Table 7). 

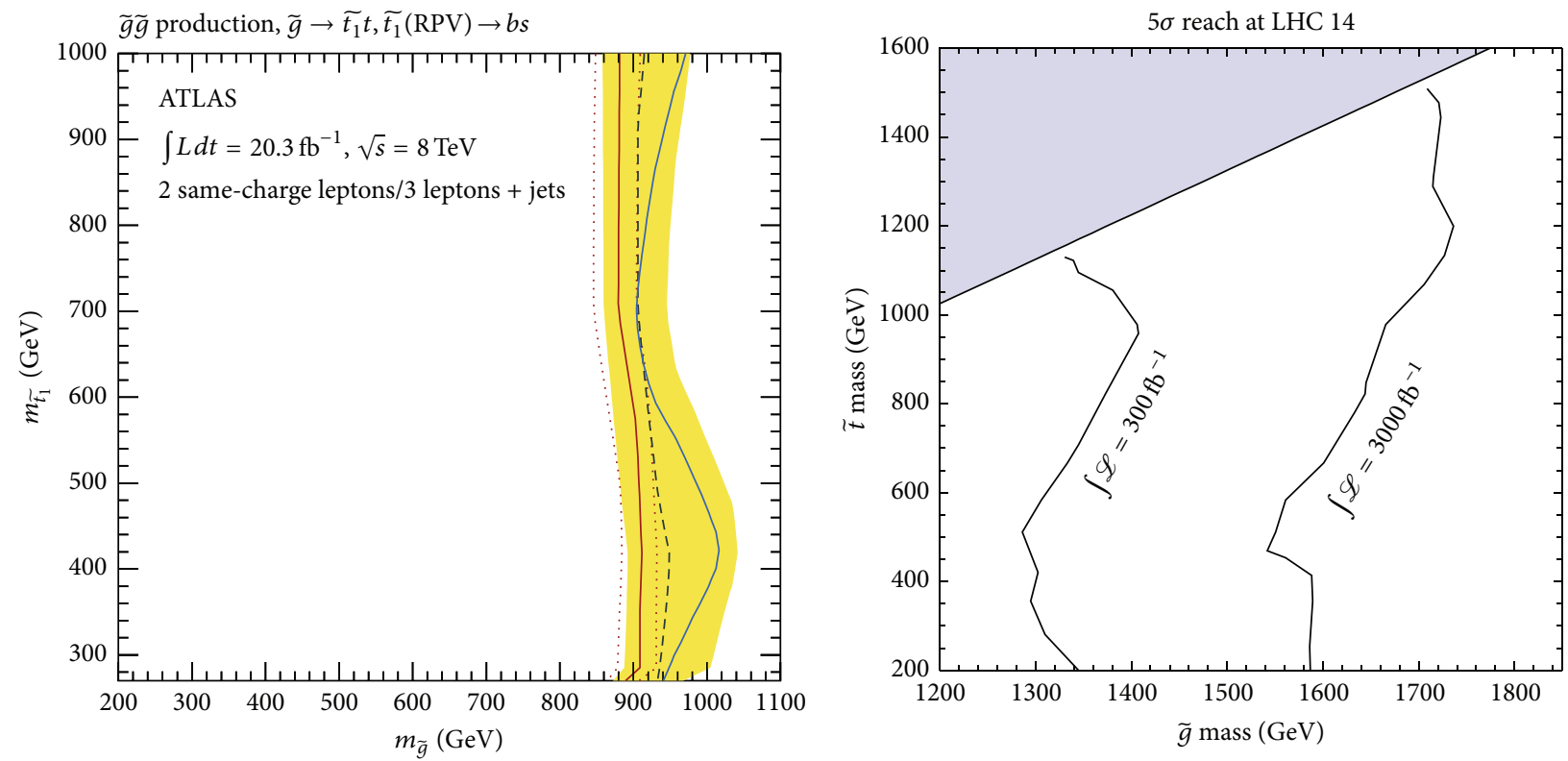

All limits at $95 \%$ CL

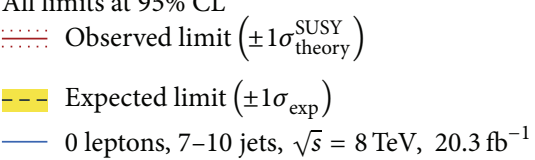

FIGURE 6: Exclusion from the LHC $8 \mathrm{TeV}$ run (a) [12] and expected discovery reach (b) [13] for the RPV gluino simplified model decaying into heavy flavors in the same-sign dilepton channel. Points at the left of the solid lines are excluded under the assumption indicated by the respective labels.

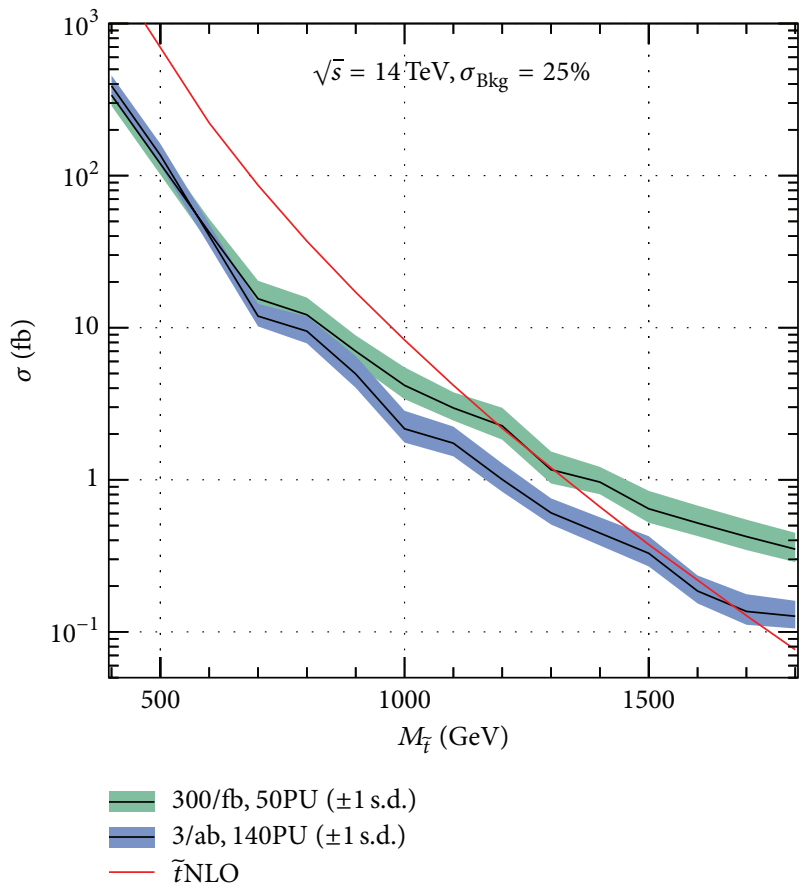

(a)

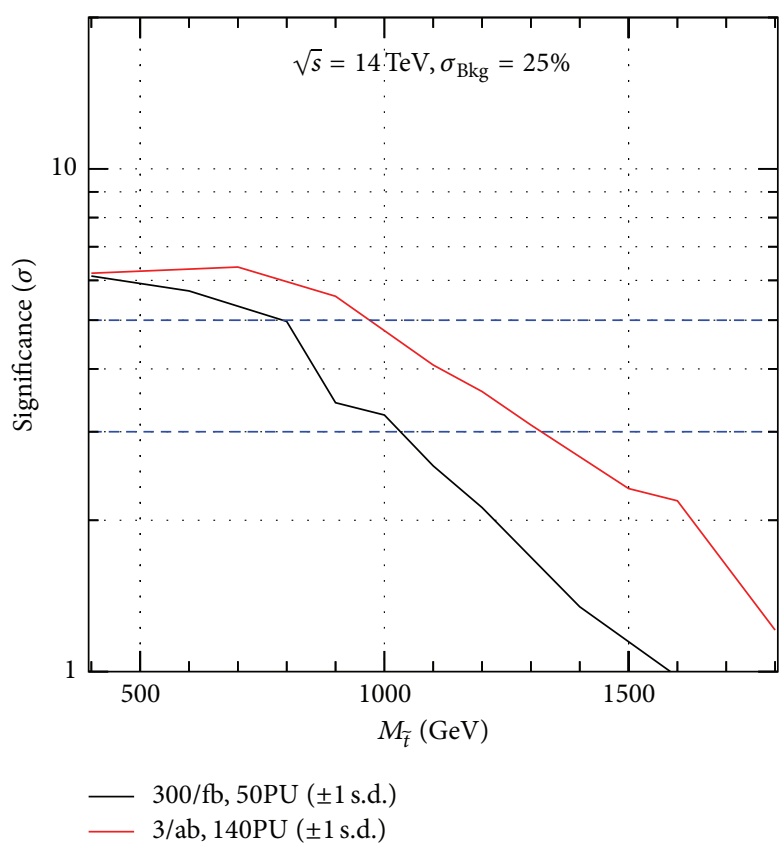

(b)

Figure 7: Exclusion (a) and discovery reach (b) for the High Luminosity LHC for the $\widetilde{t}-\chi$ RPV simplified model [6]. In the two panels, the two curves correspond to different hypothesis for integrated luminosity and average number of Pile Up (PU) collisions. 
TABLE 5: The exclusion reach of several RPV SUSY simplified models beyond those discussed in the main text [6].

\begin{tabular}{|c|c|c|c|c|c|c|}
\hline Coupling & Production & Final states & Search & $300 \mathrm{fb}^{-1}$ & $3 \mathrm{ab}^{-1}$ & $33 \mathrm{TeV}$ \\
\hline \multirow{2}{*}{ LLE122 } & $\tilde{g} / \widetilde{q} \rightarrow \widetilde{B}$ & $j j+\ell^{+} \ell^{-} \mu^{+} \mu^{-}+\not E_{T}$ & Multi- $\ell$ & 3550 & 4000 & 8500 \\
\hline & $\widetilde{W}$ & $\ell^{+} \ell^{-} \mu^{+} \mu^{-}+\not E_{T}$ & Multi- $\ell$ & 1800 & 2300 & 4400 \\
\hline \multirow{2}{*}{ LLE233 } & $\tilde{t} \rightarrow \widetilde{H}$ & $b \bar{b} \tau^{+} \tau^{-} \ell^{+} \ell^{-}+E_{T}$ & Multi- $\ell$ & 1650 & 1950 & 3750 \\
\hline & $\widetilde{H}$ & $\tau^{+} \tau^{-} \ell^{+} \ell^{-}+\not E_{T}$ & Multi- $\ell$ & 950 & 1300 & 2900 \\
\hline LQD232 & $\tilde{g} \rightarrow \tilde{t}$ & $t \bar{t}\left\{\mu^{+} j\right\}\left\{\mu^{-} j\right\}$ & Multi- $\ell$ & 2500 & 2800 & 6300 \\
\hline LQD333 & $\tilde{t}$ & $\left\{\tau^{+} b\right\}\left\{\tau^{-} b\right\}$ & 3G LQ & 1300 & 1650 & - \\
\hline UDD212 & $\tilde{t} \rightarrow \widetilde{B}$ & $t \bar{t}\{j j j\}\{j j j\}$ & $\ell+n$ jets & 1200 & 1650 & - \\
\hline UDD312 & $\widetilde{t}$ & $\{j j\}\{j j\}$ & Dijet pairs & 750 & 1070 & - \\
\hline LH3 & $\widetilde{H}$ & $W^{+} W^{-} \tau^{+} \tau^{-}$ & Multi- $\ell$ & 530 & 610 & 2800 \\
\hline
\end{tabular}

TABLE 6

\begin{tabular}{lcc}
\hline BSM particles & Production & Decay \\
\hline$\tilde{q}, \chi \quad \tilde{q} \underset{\text { prompt }}{\longrightarrow} q \chi, \chi \underset{\text { displaced }}{\stackrel{\mathrm{LQd}^{c}}{\longrightarrow} \ell j j,}$ \\
\end{tabular}

TABLE 7

\begin{tabular}{lll}
\hline $\begin{array}{l}\text { BSM } \\
\text { particles }\end{array}$ & Production & Decay \\
\hline$\chi \quad p p \rightarrow \chi \chi \quad \chi \frac{\mathrm{LQd}^{c}}{\text { displaced }} \ell j j, \chi \frac{\mathrm{LLe}^{c}}{\text { displaced }} \ell \ell \nu, \chi \frac{u^{c} d^{c} d^{c}}{\text { displaced }}, j j j$ \\
\hline
\end{tabular}

In this simplified model, there is only pure electroweak production of $\chi$, which gives rise to essentially the same displaced objects as in the case of Section 3.4.1 above. However, the cross sections are usually very small, which prevents obtaining any useful bound.

Despite these difficulties, it has been shown that the analysis [16] might have a sensitivity to this simplified model. The analysis of [16] searches for a pair of jets coming from a common displaced vertex. This signature might be relevant for $L Q d^{c}$ and $u^{c} d^{c} d^{c}$ mediated decays of the neutralino. However an interpretation of the cross section limits on these scenarios is not provided. In Figure 11, we report a recast of [16] done in [19] for models that have a long-lived particle that decays into three partons. The recast crucially depends on an accurate reproduction of the vertexing performances of CMS; therefore an interpretation from the CMS experiment would still be the preferred way to put limits on these scenarios. Nevertheless, the message from [19] is clear, and bounds up to almost $800 \mathrm{GeV}$ for Higgsinos are expected when they give rise to a displaced jet pair. Strikingly, these bounds might be the only bounds from the LHC on RPV Higgsino LSP scenario.

It should be remarked that this search for displaced jets is also sensitive to displaced decays of gluinos, which, despite hadronizing before decaying, should give rise to jets that are very similar to the ones from the electroweak neutralino considered here. Limits for the gluino scenario are reported in Figure 11. The figure also reports recast limits from [19] for large lifetimes, of the order $0.1 / \mathrm{m}$, where the displaced jets search is replaced by a search for Heavy Charged Stable
TABLE 8

\begin{tabular}{lcc}
\hline BSM particles & Production & Decay \\
\hline$\tilde{t}$ & $p p \rightarrow \widetilde{t t^{*}}$ & $\tilde{t} \underset{\text { displaced }}{\stackrel{\mathrm{LQd}^{c}}{\longrightarrow}} b \ell, \tilde{t} \underset{\text { displaced }}{\stackrel{u^{c} d^{c} d^{c}}{\longrightarrow}} j j$ \\
\hline
\end{tabular}

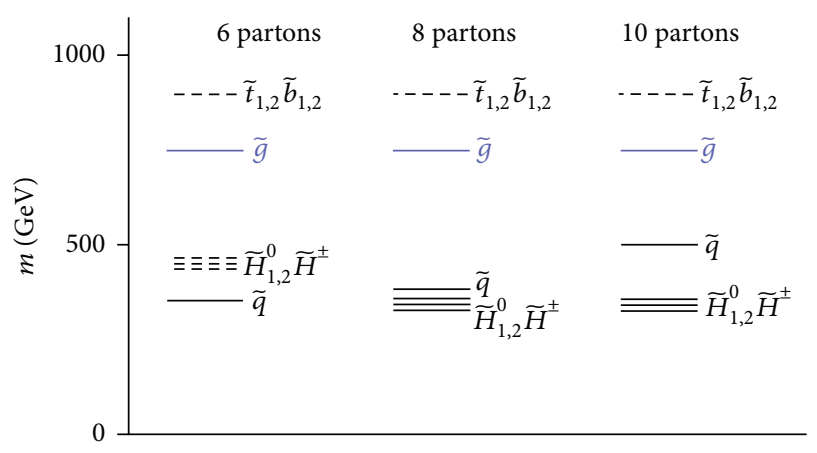

Figure 8: Possible RPV spectra that give rise to large jet multiplicity signals with little or no mET, leptons, or photons [14].

Particles [54, 55], which would be the R-hadron that forms from the long-lived gluinos.

In case of pure electroweak production, the very clean $L L e^{c}$ decay into leptons might give a signal in the analysis for displaced same-flavor lepton pairs [18], but at present there is no such interpretation.

3.4.3. The Displaced RPV Squark Simplified Model. For this simplified model, it is assumed that only squarks are light enough to be produced (see Table 8).

A search by CMS [20] has used this model to put bounds on final states where two different flavors leptons from $L Q d^{c}$ interaction emerge both with large impact parameter. The results are given in Figure 12 and bounds up to about $800 \mathrm{GeV}$ for one stop squark are obtained.

The selection of the analysis [20] is very inclusive, as it targets a final state $e \mu+X$ and no special requirements are imposed on $X$. For this reason, one could expect that significant bounds can be extracted for light flavor squarks instead of stops decaying to $b$ quarks, $p p \rightarrow \widetilde{q} \widetilde{q} \rightarrow q \mu q e$, and for more complex signatures such as those from the displaced neutralino studied in previous Sections 3.4.1 and 3.4.2. 


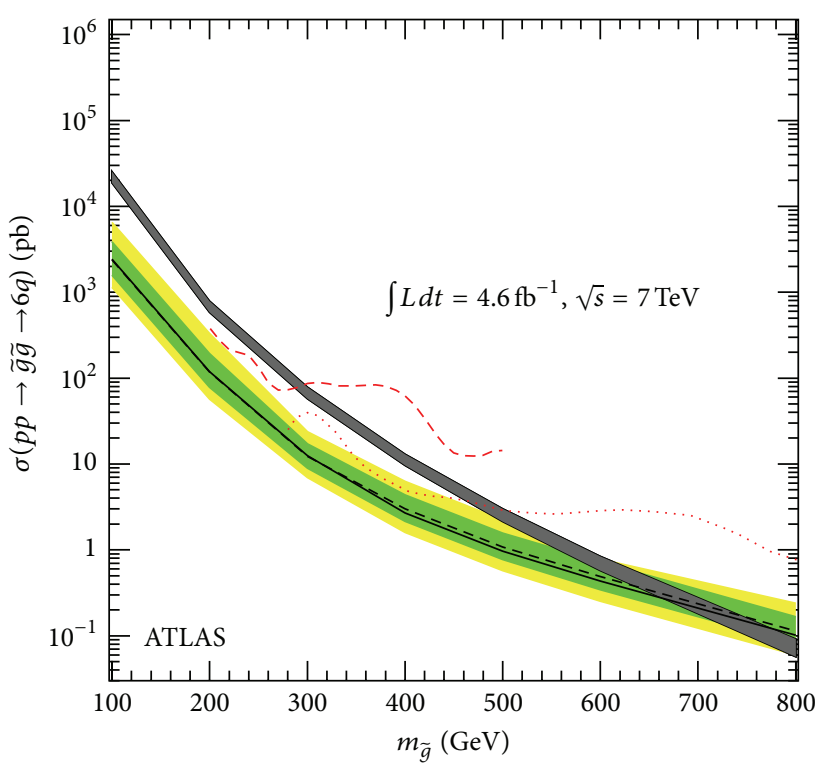

All limits at 95\% CL

- - - Expected limit (resolved)

_ Observed limit (resolved)

- - - Observed limit (CMS 2010)

Observed limit (CMS 2011)

(a) $\pm 2 \sigma$ Expected limit (resolved)

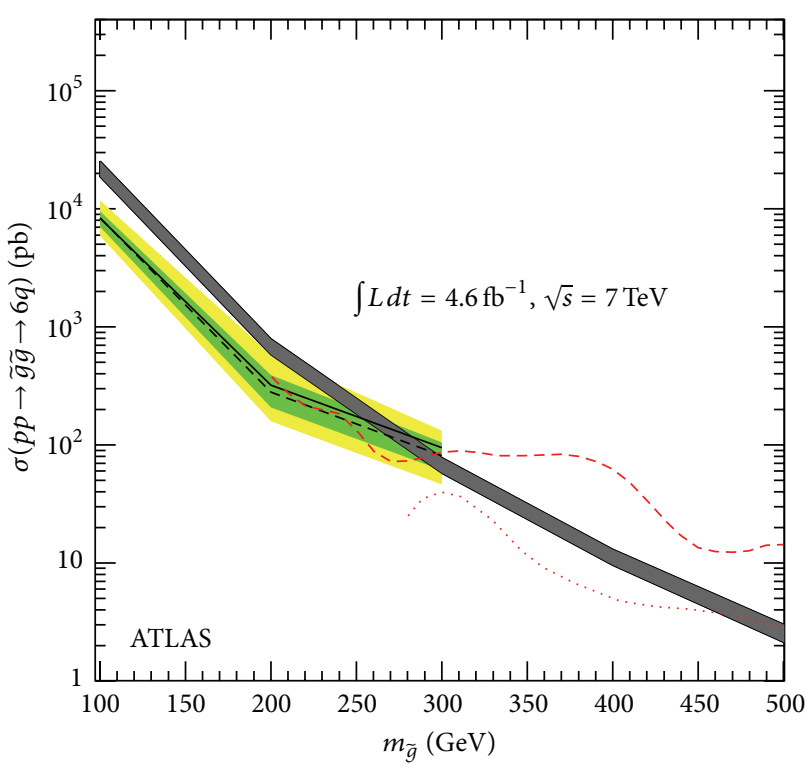

All limits at 95\% CL

- - - Expected limit (resolved)

_ Observed limit (resolved)

- - - Observed limit (CMS 2010)

.. Observed limit (CMS 2011)

$\pm 1 \sigma$ Expected limit (resolved) $\pm 2 \sigma$ Expected limit (resolved)

$\tilde{g} \widetilde{g}$ cross section (NLO + NLL)

(b)

FIGURE 9: Exclusion of a search in multijet final state (a) and a boosted resonance search with fat jets (b) on the same 7 TeV data set of ATLAS [15]. Color codes as in Figure 1.

For hadronic final states that emerge from squark decay mediated by the $u^{c} d^{c} d^{c}$ interaction, there is no explicit interpretation from the experiments searching for displaced jets; however, as in the cases discussed above, bounds are expected from a displaced jet search such as [16]. The recast of this search proposed by [19] is shown in Figure 13 (together with a typical event display of two largely displaced jets). The results of the recast show clear potential of exclusion for masses up to $1 \mathrm{TeV}$.

When the RPV couplings are small enough, the squarks will decay only after they have formed hadrons. If the squark is sufficiently long-lived it is best to search for the hadrons produced in its hadronization. In fact, among the hadrons formed, one should be very massive compared to ordinary hadrons and might also be electrically charged. The search for massive stable charged particles has been carried out at the LHC [54, 55] and these limits have also been recast [19] for the scenario of R-hadrons from squark hadronization. The result of this recast is shown in Figure 13, which suggests that squark masses up to $900 \mathrm{GeV}$ should be ruled out by this type of searches.

\section{Conclusions}

After the first run of the LHC, the most striking signatures of $\mathrm{TeV}$-scale supersymmetric particles have not been observed. The bounds on colored superparticles are particularly stringent and start to seriously challenge the paradigm of minimal models of supersymmetry at the weak scale.
Several mechanisms to avoid LHC bounds have been identified as a reaction to the results of the first run of the experiments. Among the possible ideas to alleviate this tension between supersymmetry and experiments, the possibility of a violation of R-parity emerges as a conceptually very simple and motivated option. Furthermore in recent times a new wave of works on the origin of R-parity breaking couplings $[31,32,56]$ has provided new ways to formulate predictive and realistic supersymmetric model without Rparity.

In addition to the results coming from the direct exploration of the $\mathrm{TeV}$ scale at particle colliders, the results from Dark Matter searches are also shedding new light on the conservation of R-parity in supersymmetric models. In fact direct searches for weakly interacting massive particles have already excluded large portions of the parameter space of the minimal models with conserved R-parity. This motivates alternative scenarios for Dark Matter, which could easily fit in R-parity violating models, where light and very weakly interacting particles naturally emerge as Dark Matter candidates.

The new directions suggested by the results of the LHC experiments and Dark Matter searches both make supersymmetric models with broken R-parity a very motivated scenario for new physics at the $\mathrm{TeV}$ scale.

On top of these motivations from experimental and theoretical considerations, it is remarkable that the breaking of R-parity significantly enlarges the set of experimental signatures of new physics. Therefore one can add a more pragmatic motivation to study R-parity violating models as "signatures 


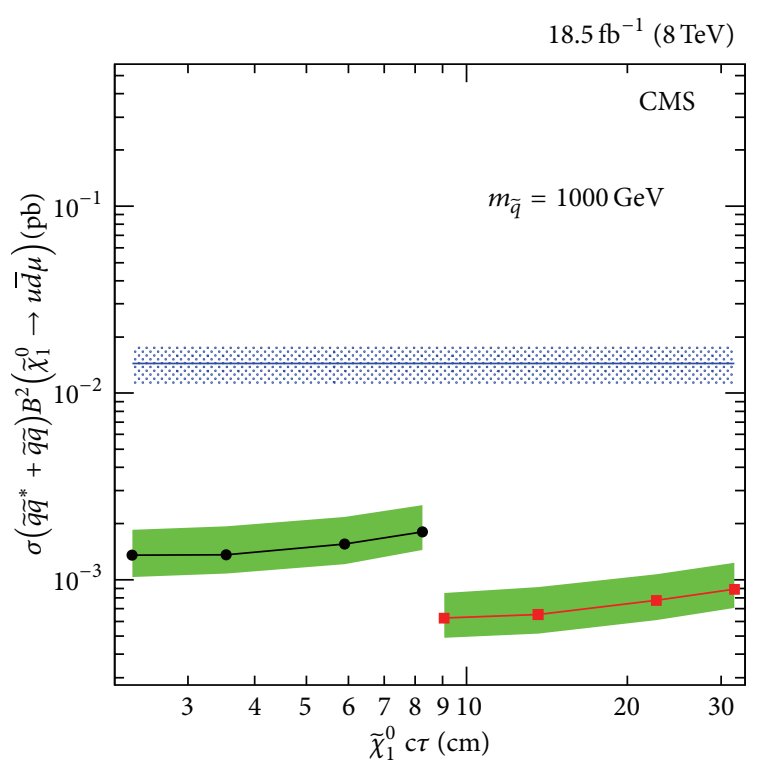

95\% CL limits:

$$
\begin{array}{ll}
\rightarrow m_{\tilde{\chi}_{1}^{0}}=150 \mathrm{GeV} & \square \text { Expected limits }( \pm 1 \sigma) \\
\rightarrow-m_{\tilde{\chi}_{1}^{0}}=500 \mathrm{GeV} & \sigma_{\tilde{q}^{*}+\tilde{q} \tilde{q}} \pm 1 \sigma_{\text {theory }}
\end{array}
$$

(a)

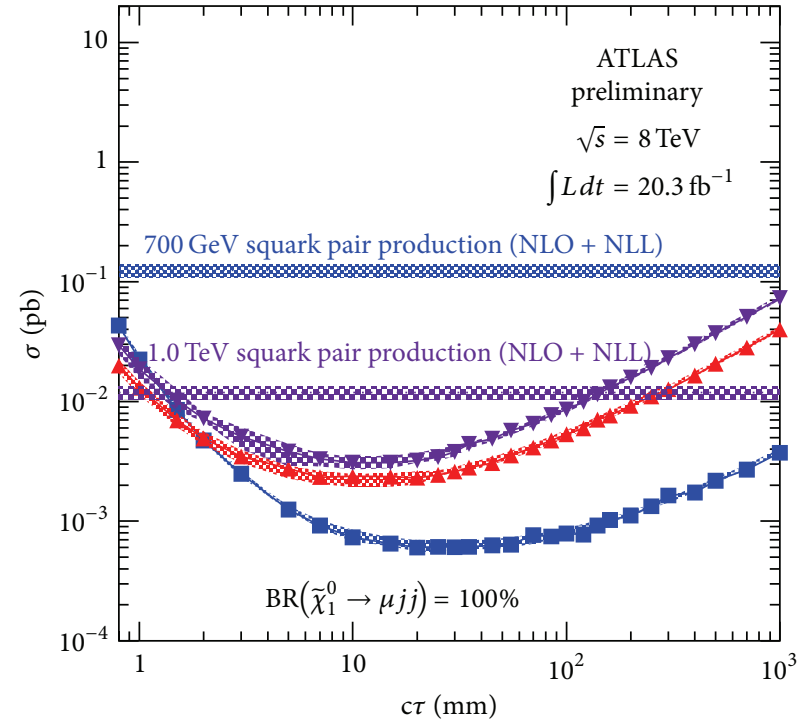

$\mathrm{MH}(700,494)$

. ML $(700,108)$

HL $(1000,108)$

(b)

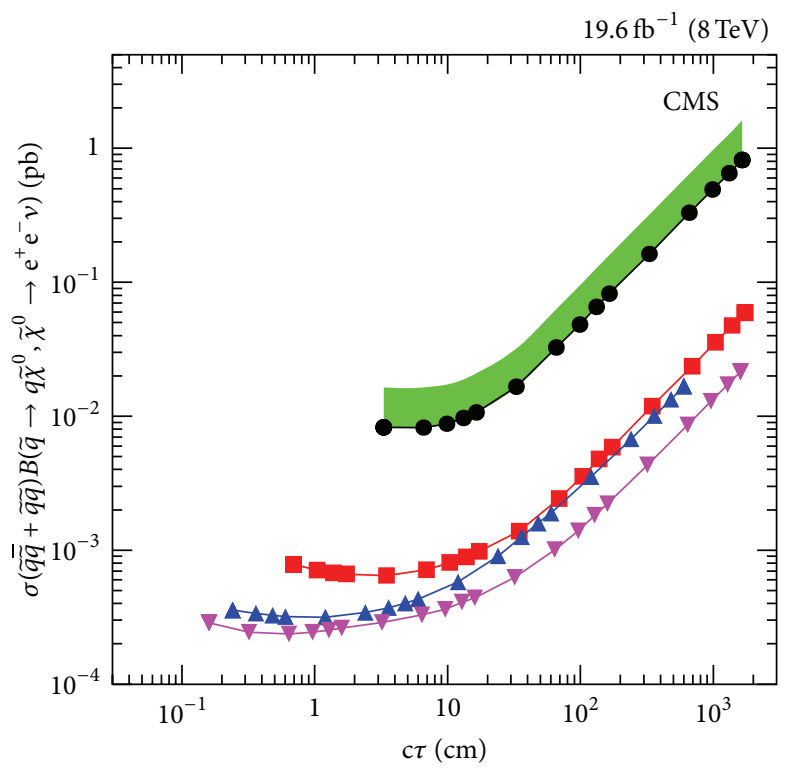

$$
\begin{array}{lll}
\text { Observed limits } & m_{\tilde{q}} / m_{\tilde{\chi}}=1500 / 494 \mathrm{GeV} / c^{2} \\
\rightarrow m_{\tilde{q}} / m_{\tilde{\chi}}=120 / 48 \mathrm{GeV} / c^{2} & \text { Expected limits }( \pm 1 \sigma) \\
\rightarrow-m_{\tilde{q}} / m_{\tilde{\chi}}=350 / 148 \mathrm{GeV} / c^{2} & m_{\tilde{q}} / m_{\tilde{\chi}}=120 / 48 \mathrm{GeV} / c^{2} \\
\leftarrow m_{\tilde{q}} / m_{\tilde{\chi}}=1000 / 148 \mathrm{GeV} / c^{2} &
\end{array}
$$

(c)

FIGURE 10: Limits on displaced RPV supersymmetry for LQD coupling from displaced jets [16] (a), displaced muon + tracks events [17] (b), and for LLE coupling from displaced same-flavor dileptons [18] (c). In all panels, each colored line represents an example of new physics spectrum for which the cross section indicated in the vertical axis is excluded at $95 \%$ Confidence Level under the assumption of the lifetime indicated in the corresponding horizontal axis. The horizontal lines are predictions for the total production cross section in new physics scenarios for new physics particles masses as labeled on each panel. 

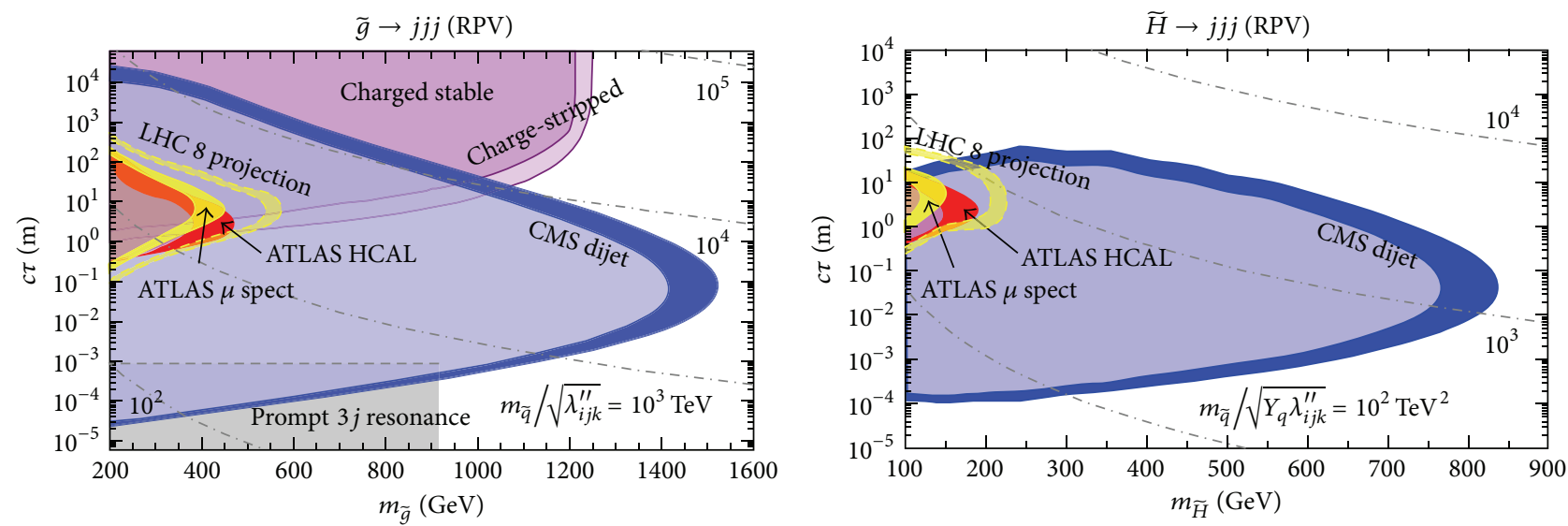

FIGURE 11: Limits on baryonic RPV from displaced jets searches [16] recast in [19]. For each type of search, a colored area indicated the excluded region in the plane lifetime-mass of the metastable new state. The gray area corresponds to the exclusion from searches for prompt (i.e., nondisplaced) new physics, which is substantially weaker than what can be achieved by most displaced searches. For each color, the darker band represents the uncertainty on the excluded area that comes from varying the reconstruction efficiency for displaced objects.

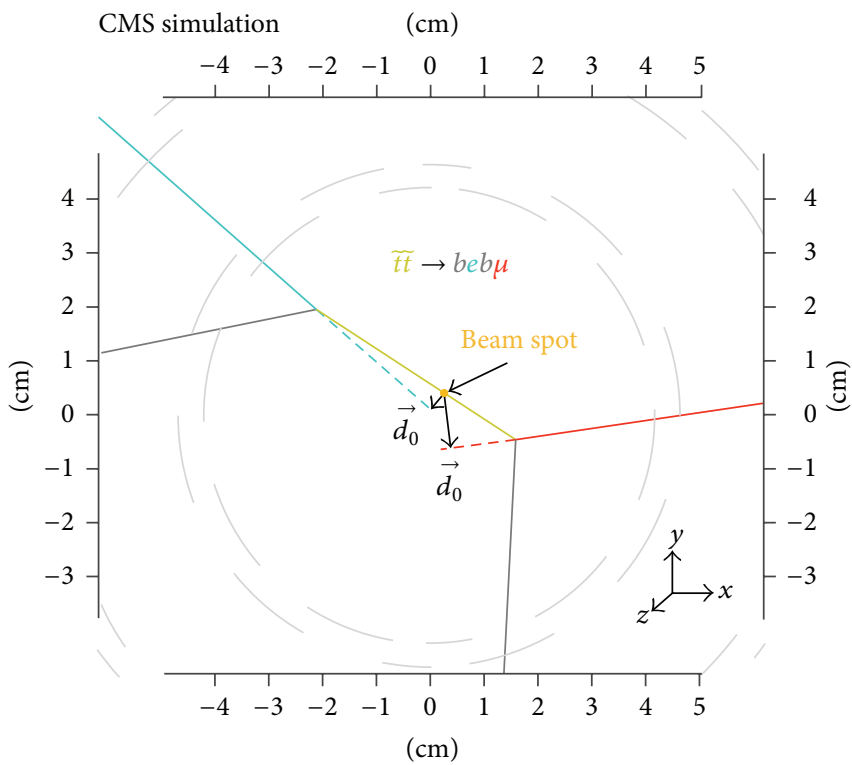

(a)

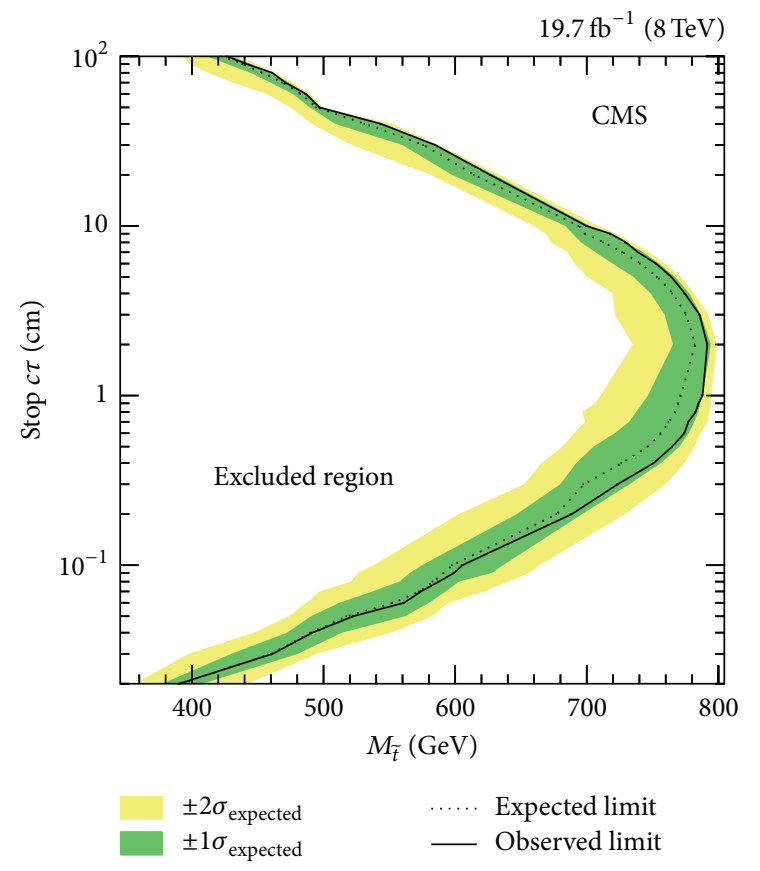

(b)

FIGURE 12: Limits on displaced RPV supersymmetry for stops decaying with equal rates into the three leptonic flavors [20]. On panel (a), a sample schematic event display is shown. The panel (b) gives the exclusion in the stop lifetime-mass plane. Points to the left of the solid black curve are excluded. Color codes as in Figure 1.

generators," whose investigation further widens the scope of the search for new physics at the LHC.

In this work, we have presented the large body of results available on the searches for new physics that has been interpreted (or can be relevant) for R-parity violating scenarios. In this large set of results, two examples of the extended coverage for signals of new physics that has been brought to the attention of the experiments by the study of R-parity violating models are multijet resonant signatures and displaced signatures. The latter are a rather interesting example.
In fact, the presence of displaced objects (tracks, leptons, jets, etc.) in an event is difficult to deal with at the experimental level. Nevertheless, the experimental difficulties have been overcome and these signatures in many cases provide the most stringent mass bounds on the existence of certain types of new particles. In other cases, such as new particles that decay promptly to many jets, large improvement has been obtained thanks to the input from the phenomenology of R-parity violating models. For instance, the presence of heavy flavor tags has gained importance in resonant multijet 


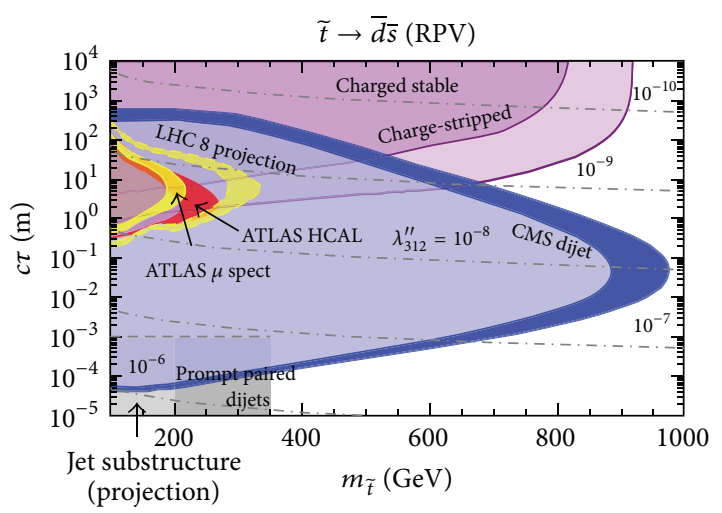

(a)

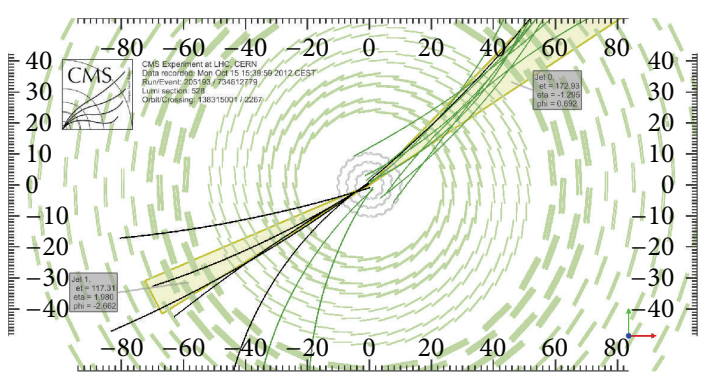

(b)

Figure 13: Results on limits on displaced jets searches [16] recast for baryonic RPV decays of squarks [19] (a). An event display [16] of the tracks that make a jet pair event from a displaced vertex (b). The yellow cones are the two jets making the pair and the black tracks are those fitting to a secondary vertex displaced by $5 \mathrm{~cm}$. Same notation as in Figure 11.

searches. Furthermore, the study of these signals has been useful because it has shown the difficulties that LHC experiments encounter when searching for such signals, thus calling for the development of new strategies to search for new physics.

The large amount of possible hierarchies of RPV couplings and the several motivated spectra of the new particles produce a large set of possible signals, some of which is yet to be captured by the analyses of the LHC experiments, which leaves more work to be done in the future both in including new signatures in LHC searches and in developing more refined ways to highlight new physics signals from the data.

\section{Conflict of Interests}

The author declares that there is no conflict of interests regarding the publication of this paper.

\section{Acknowledgments}

It is a pleasure to thank Freya Blekman, Dinko Ferenček, Giacomo Polesello, Andrea Romanino, Prashant Saraswat, and Brock Tweedie for stimulating discussions and many inputs on these topics. The author also would like to thank Gabriele Ferretti, Rabindra Mohapatra, Christoffer Petersson, and Riccardo Torre for many discussions and the collaboration for the papers that the author has coauthored. The author would like to thank Andrea Thamm for very useful comments on the paper.

\section{References}

[1] B. Nachman and T. Rudelius, "Evidence for conservatism in LHC SUSY searches," The European Physical Journal Plus, vol. 127, article 157, 2012.

[2] CMS Collaboration, "SUSY searches summary plots," https:// twiki.cern.ch/twiki/bin/view/CMSPublic/PhysicsResultsSUS.
[3] W. Pauli, "Open letter to the group of radioactive people at the Gauverein meeting in Tübingen," http://microboonedocdb.fnal.gov/cgi-bin/RetrieveFile?docid=953; filename=pauli \%20letter1930.pdf; version=1.

[4] T. Aaltonen, E. Albin, S. Amerio et al., "Search for pair production of strongly interacting particles decaying to pairs of jets in $p \bar{p}$ collisions at $\sqrt{s}=1.96 \mathrm{TeV}$," Physical Review Letters, vol. 111, Article ID 031802, 2013.

[5] CMS Collaboration, "Search for pair-produced resonances decaying to jet pairs in proton-proton collisions at $\sqrt{s}=8 \mathrm{TeV}$," Physics Letters B, vol. 747, pp. 98-119, 2015.

[6] D. Duggan, J. A. Evans, J. Hirschauer et al., "Sensitivity of an upgraded LHC to R-parity violating signatures of the MSSM," http://arxiv.org/abs/1308.3903.

[7] T. Aaltonen, B. A. González, S. Amerio et al., "First search for multijet resonances in $\sqrt{s}=1.96 \mathrm{TeV} p \bar{p}$ collisions," Physical Review Letters, vol. 107, no. 4, Article ID 042001, 2011.

[8] S. Chatrchyan, V. Khachatryan, A. M. Sirunyan et al., "Search for three-jet resonances in $p p$ collisions at $\sqrt{s}=7 \mathrm{TeV}$," Physical Review Letters, vol. 107, no. 10, Article ID 101801, 15 pages, 2011.

[9] CMS Collaboration, "Search for three-jet resonances in $p p$ collisions at $\sqrt{s}=7$ TeV," Physics Letters B, vol. 718, pp. 329-347, 2012.

[10] CERN, "Search for light- and heavy-flavor three-jet resonances in multijet final states at 8 tev," Tech. Rep. CMS-PAS-EXO-12049, CERN, Geneva, Switzerland, 2013, https://twiki.cern.ch/ twiki/bin/view/CMSPublic/PhysicsResultsEXO12049.

[11] ATLAS Collaboration, "Search for massive particles in multijet signatures with the ATLAS detector in $\sqrt{s}=8 \mathrm{TeV}$ pp collisions at the LHC,' Tech. Rep. ATLAS-CONF-2013-091, CERN, Geneva, Switzerland, 2013, https://atlas.web.cern.ch/Atlas/GROUPS/ PHYSICS/CONFNOTES/ATLAS-CONF-2013-091/.

[12] ATLAS Collaboration, "Search for supersymmetry at $N \sqrt{s}=$ $8 \mathrm{TeV}$ in final states with jets and two same-sign leptons or three leptons with the ATLAS detector," Journal of High Energy Physics, vol. 2014, article 35, 2014.

[13] M. Saelim and M. Perelstein, "RPV SUSY with same-sign dileptons at LHC-14," http://arxiv.org/abs/1309.7707. 
[14] J. A. Evans, Y. Kats, D. Shih, and M. J. Strassler, "Toward full LHC coverage of natural supersymmetry," Journal of High Energy Physics, vol. 2014, no. 7, article 101, 2014.

[15] ATLAS Collaboration, "Search for pair production of massive particles decaying into three quarks with the ATLAS detector in $\sqrt{s}=7 \mathrm{TeV}$ pp collisions at the LHC, Journal of High Energy Physics, vol. 2012, no. 12, article 86, 2012.

[16] CMS Collaboration, "Search for long-lived neutral particles decaying to quark-antiquark pairs in proton-proton collisions at $\sqrt{s}=8 \mathrm{TeV}$," Physical Review D, vol. 91, no. 1, Article ID 012007, 21 pages, 2014.

[17] CERN, "Search for long-lived, heavy particles in final states with a muon and a multi-track displaced vertex in protonproton collisions at $\sqrt{s}=8 \mathrm{TeV}$ with the ATLAS detector," Tech. Rep. ATLAS-CONF-2013-092, CERN, Geneva, Switzerland, 2013, https://atlas.web.cern.ch/Atlas/GROUPS/PHYSICS/ CONFNOTES/ATLAS-CONF-2013-092/.

[18] V. Khachatryan, A. M. Sirunyan, A. Tumasyan et al., "Search for long-lived particles that decay into final states containing two electrons or two muons in proton-proton collisions at $\sqrt{s}=8$ TeV," Physical Review D, vol. 91, Article ID 052012, 2015.

[19] Z. Liu and B. Tweedie, "The fate of long-lived superparticles with hadronic decays after LHC Run 1," Journal of High Energy Physics, vol. 2015, article 42, 2015.

[20] V. Khachatryan, A. M. Sirunyan, A. Tumasyan et al., "Search for displaced supersymmetry in events with an electron and a muon with large impact parameters," Physical Review Letters, vol. 114, Article ID 061801, 2014.

[21] R. Barbier, C. Bérat, M. Besançon et al., "R-Parity-violating supersymmetry," Physics Reports, vol. 420, no. 1-6, pp. 1-195, 2005.

[22] W. Buchmüller, L. Covi, K. Hamaguchi, A. Ibarra, and T. T. Yanagida, "Gravitino dark matter in $R$-parity breaking vacua," Journal of High Energy Physics, vol. 3, article 37, 2007.

[23] F. Takayama and M. Yamaguchi, "Gravitino dark matter without R-parity," Physics Letters B, vol. 485, no. 4, pp. 388-392, 2000.

[24] F. D. Steffen, "Gravitino dark matter and cosmological constraints," Journal of Cosmology and Astroparticle Physics, vol. 2006, no. 9, article 001, 2006.

[25] R. Franceschini and R. N. Mohapatra, "New patterns of natural $R$-parity violation with supersymmetric gauged flavor," Journal of High Energy Physics, vol. 2013, no. 4, article 98, 2013.

[26] C. Csáki, Y. Grossman, and B. Heidenreich, "Minimal flavor violation supersymmetry: a natural theory for $R$-parity violation," Physical Review D, vol. 85, no. 9, Article ID 095009, 2012.

[27] J. Fan, M. Reece, and J. T. Ruderman, "A stealth supersymmetry sampler," Journal of High Energy Physics, vol. 2012, no. 7, article 196, 2012.

[28] P. Graham, S. Rajendran, and P. Saraswat, "Supersymmetric crevices: missing signatures of $R$-parity violation at the LHC," Physical Review D, vol. 90, Article ID 075005, 2014.

[29] ATLAS Collaboration, "Search for pair-produced massive coloured scalars in four-jet final states with the ATLAS detector in proton-proton collisions at $\sqrt{s}=7 \mathrm{TeV}$," The European Physical Journal C, vol. 73, article 2263, 2013.

[30] Y. Bai, A. Katz, and B. Tweedie, "Pulling out all the stops: searching for RPV SUSY with stop-jets," Journal of High Energy Physics, vol. 2014, no. 1, article 040, 2014.

[31] C. Smith, "Minimal flavor violation as an alternative to Rparity", in Proceedings of the 34th International Conference on High Energy Physics (ICHEP '08), Philadelphia, Pa, USA, 2008.
[32] C. Csaki, Y. Grossman, and B. Heidenreich, "MFV SUSY: a natural theory for R-parity violation," Physical Review D, vol. 85, no. 9, Article ID 095009, 22 pages, 2012.

[33] G. Krnjaic and D. Stolarski, "Gauging the way to MFV," Journal of High Energy Physics, vol. 2013, article 64, 2013.

[34] C. Csáki and B. Heidenreich, "A complete model for R-parity violation," Physical Review D, vol. 88, Article ID 055023, 2013.

[35] R. Franceschini and R. Torre, "RPV stops bump off the background," The European Physical Journal C, vol. 73, article 2422, 2013.

[36] I. Hinchliffe, F. E. Paige, M. D. Shapiro, J. Söderqvist, and W. Yao, "Precision SUSY measurements at CERN LHC", Physical Review D, vol. 55, pp. 5520-5540, 1997.

[37] B. C. Allanach, C. Gorham Lester, M. A. Parker, and B. R. Webber, "Measuring sparticle masses in non-universal string inspired models at the LHC," Journal of High Energy Physics, vol. 2000, no. 9, article 004, 2000.

[38] K. Kawagoe, M. M. Nojiri, and G. Polesello, "New supersymmetry mass reconstruction method at the CERN LHC," Physical Review D-Particles, Fields, Gravitation and Cosmology, vol. 71, no. 3, Article ID 35008, 2005.

[39] F. W. Stecker, "Cosmic gamma rays," NASA Special Publication 249, 1971.

[40] K. Agashe, R. Franceschini, and D. Kim, "Simple 'invariance' of two-body decay kinematics," Physical Review D, vol. 88, no. 5, Article ID 057701, 2013.

[41] J. Eckel, W. Shepherd, and S. Su, "Slepton discovery in electroweak cascade decay," Journal of High Energy Physics, vol. 2012, no. 5, article 81, 2012.

[42] I. Low, "Polarized charginos (and tops) in stop decays," Physical Review D, vol. 88, Article ID 095018, 2013.

[43] ATLAS Collaboration, "Search for a heavy narrow resonance decaying to $e \mu, e \tau$, or $\mu \tau$ with the ATLAS detector in View the MathML sourcepp collisions at the LHC," Physics Letters B, vol. 723, no. 1-3, pp. 15-32, 2013.

[44] G. Aad, B. Abbott, J. Abdallah et al., "Search for supersymmetry in events with four or more leptons in $\sqrt{s}=8 \mathrm{TeV} p p$ collisions with the ATLAS detector," Physical Review D, vol. 90, Article ID 052001, 2014.

[45] ATLAS Collaboration, "Search for massive colored scalars in four-jet final states in $\sqrt{s}=7 \mathrm{TeV}$ proton-proton collisions with the ATLAS detector," The European Physical Journal C, vol. 71, article 1828, 2011.

[46] ATLAS Collaboration, "Search for new phenomena in final states with large jet multiplicities and missing transverse momentum at $\sqrt{s}=8 \mathrm{TeV}$ proton-proton collisions using the ATLAS experiment," Journal of High Energy Physics, vol. 2013, no. 10, article 130, 2013.

[47] H. Baer, C.-H. Chen, and X. Tata, "Impact of hadronic decays of the lightest neutralino on the reach of the CERN LHC," Physical Review D-Particles, Fields, Gravitation and Cosmology, vol. 55, no. 3, pp. 1466-1470, 1997.

[48] CMS Collaboration, "Search for multijet resonances in the 8-jet final state," Tech. Rep. CMS-PAS-EXO-11-075, 2012, http://cds.cern.ch/record/1482131?ln=en.

[49] S. Chatrchyan, V. Khachatryan, A. M. Sirunyan et al., "Search for microscopic black holes in pp collisions at $\sqrt{s}=8 \mathrm{TeV}$," Journal of High Energy Physics, vol. 2013, no. 7, article 178, 2013.

[50] T. Cohen, E. Izaguirre, M. Lisanti, and H. K. Lou, "Jet substructure by accident," Journal of High Energy Physics, vol. 2013, article 161, 2013. 
[51] T. Cohen, M. Jankowiak, M. Lisanti, H. K. Lou, and J. G. Wacker, "Jet substructure templates: data-driven QCD backgrounds for fat jet searches," Journal of High Energy Physics, vol. 2014, no. 5, article 005, 2014.

[52] S. El Hedri, A. Hook, M. Jankowiak, and J. G. Wacker, "Learning how to count: a high multiplicity search for the LHC", Journal of High Energy Physics, vol. 2013, no. 8, article 136, 2013.

[53] P. W. Graham, D. E. Kaplan, S. Rajendran, and P. Saraswat, "Displaced supersymmetry," Journal of High Energy Physics, vol. 2012, no. 7, article 149, 2012.

[54] CMS Collaboration, "Searches for long-lived charged particles in pp collisions at $\sqrt{s}=7$ and $8 \mathrm{TeV}$," Journal of High Energy Physics, vol. 2013, article 122, 2013.

[55] G. Aad, B. Abbott, J. Abdallah et al., "Searches for heavy longlived charged particles with the ATLAS detector in protonproton collisions at $\sqrt{s}=8 \mathrm{TeV}$,' Journal of High Energy Physics, vol. 2015, no. 1, article 068, 2015.

[56] C. Csáki, E. Kuflik, and T. Volansky, "Dynamical R-parity violation," Physical Review Letters, vol. 112, no. 13, Article ID 131801, 2014. 

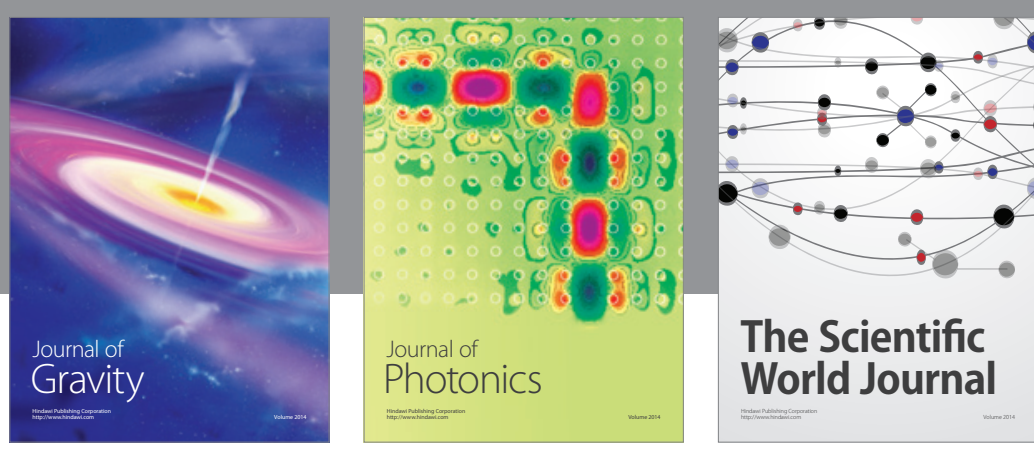

The Scientific World Journal
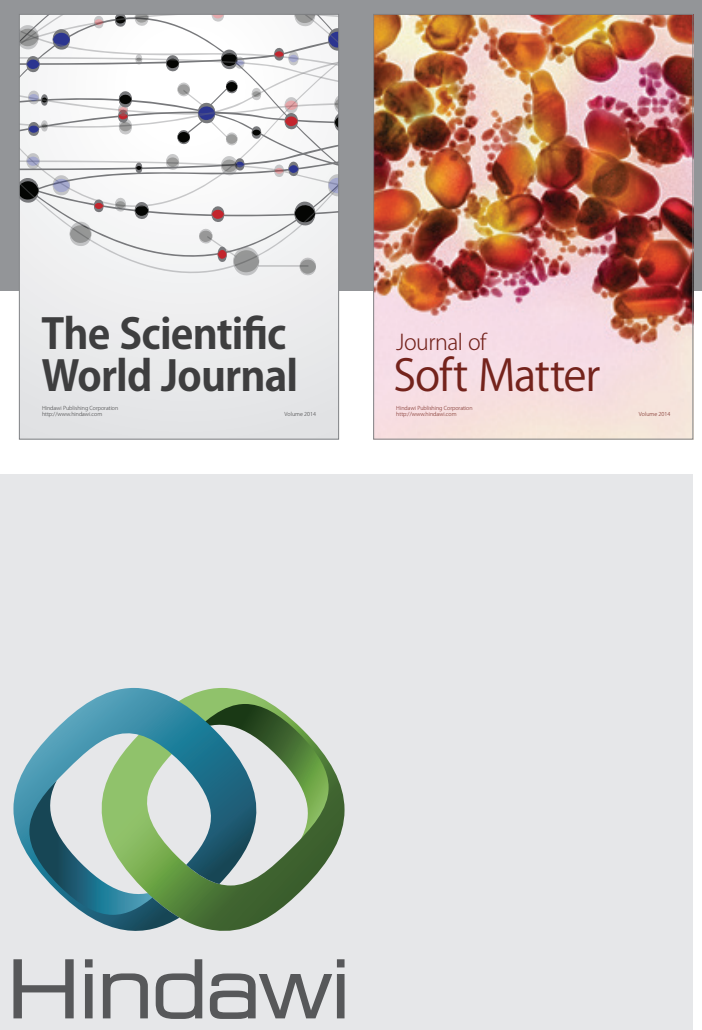

Submit your manuscripts at

http://www.hindawi.com

nternational Journal of

Statistical Mechanics
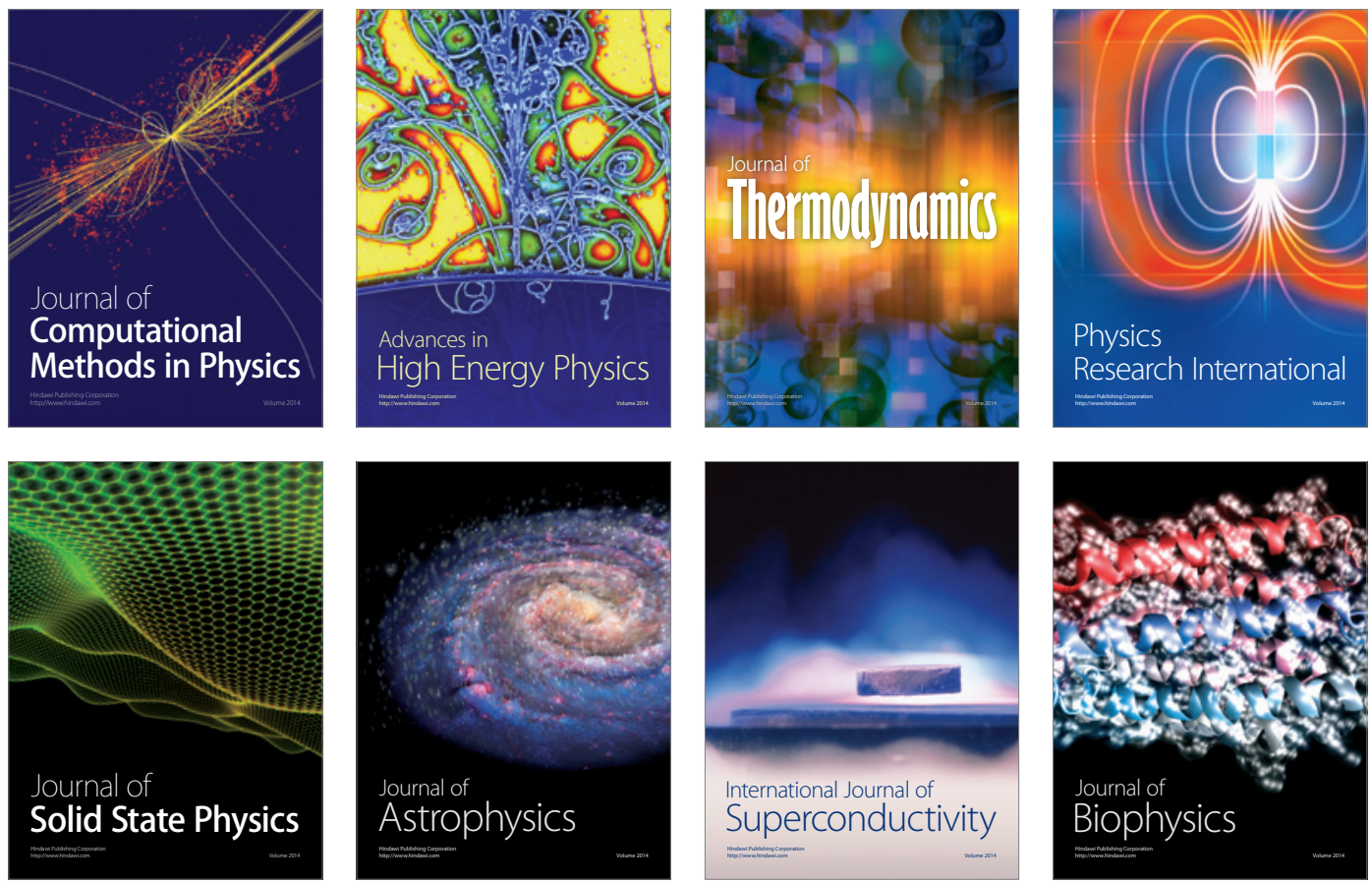
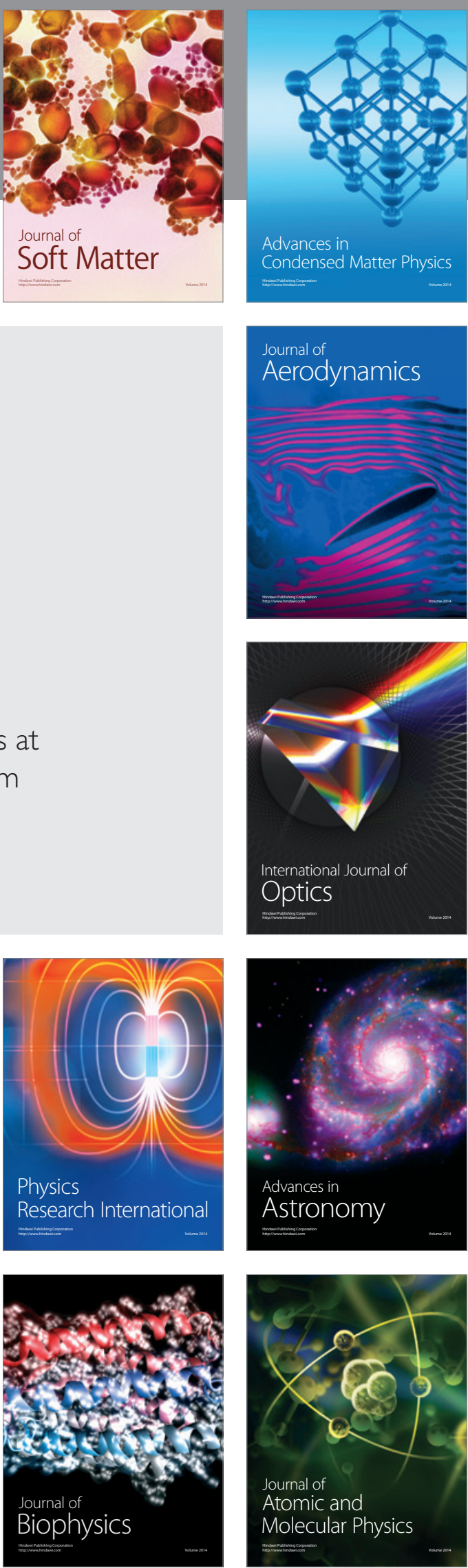